\title{
ARTICLE OPEN Jumonji domain-containing 6 (JMJD6) identified as a potential therapeutic target in ovarian cancer
}

\author{
Heng Zheng ${ }^{1}$, Yan Tie ${ }^{2}$, Zhen Fang $\mathbb{D}^{2}{ }^{2}$, Xiaoai $W^{2}{ }^{2}$, Tao Yi ${ }^{1}$, Shuang Huang ${ }^{1}$, Xiao Liang ${ }^{1}$, Yanping Qian ${ }^{1}, \mathrm{Xi} \mathrm{Wang}^{1}$, Ruyu Pi ${ }^{1}$,
} Siyuan Chen ${ }^{2}$, Yong Peng ${ }^{2}{ }^{2}$, Shengyong Yang ${ }^{2}$, Xia Zhao ${ }^{1}$ and Xiawei Wei ${ }^{2}$

Jumonji domain-containing 6 (JMJD6) is a candidate gene associated with tumorigenesis, and JMJD6 overexpression predicts poor differentiation and unfavorable survival in some cancers. However, there are no studies reporting the expression of JMJD6 in ovarian cancer, and no JMJD6 inhibitors have been developed and applied to targeted cancer therapy research. In the present study, we found that the high expression of JMJD6 in ovarian cancer was correlated with poor prognosis in ovarian cancer. A potential inhibitor (SKLB325) was designed based on the crystal structure of the jmjC domain of JMJD6. This molecule significantly suppressed proliferation and induced apoptosis in a dose-dependent manner in SKOV3 cell lines as detected by CCK-8 cell proliferation assays and flow cytometry. A Matrigel endothelial tube formation assay showed that SKLB325 inhibited capillary tube organization and migration in HUVECs in vitro. We also observed that JMJD6 colocalized with p53 protein in the nucleus, with mRNA and protein expression of $\mathrm{p} 53$ as well as its downstream effectors significantly increasing both in vitro and in intraperitoneal tumor tissues treated with SKLB325. In addition, SKLB325 significantly reduced the intraperitoneal tumor weight and markedly prolonged the survival of tumor-bearing mice. Taken together, our findings suggest that JMJD6 may be a marker of poor prognosis in ovarian cancer and that SKLB325 may be a potential candidate drug for the treatment of ovarian cancer.

Signal Transduction and Targeted Therapy (2019)4:24; https://doi.org/10.1038/s41392-019-0055-8

\section{BACKGROUND}

Ovarian cancer is the principal cause of death among gynecological malignant tumors. ${ }^{1-3}$ Mortality remains extraordinarily high, despite many studies on the mechanism of ovarian cancer ${ }^{4}$ and many improvements in treatments for patients with ovarian cancer, including surgery, radiotherapy, chemotherapy and new biological therapies. ${ }^{5-7}$ In addition, more than $70 \%$ of ovarian cancer patients are at advanced stages when initially diagnosed, and the 5 -year survival rate is less than $30 \% .^{8}$ This terrible situation reflects a lack of novel, effective and feasible therapeutic approaches to treat ovarian cancer.

JMJD6, a member of the Jumonji C (JMJC) domain-containing family of proteins, which is a lysine hydroxylase, ${ }^{9,10}$ may regulate the transcriptional activity of $\mathrm{p} 53$ by hydroxylation of a lysine in the p53 C-terminal structural domain. ${ }^{11-14}$ Initially, JMJD6 was studied as the phospholipid methionine receptor on the surface of phagocytic cells. ${ }^{15-17}$ Subsequently, many studies showed that JMJD6 has multiple nuclear positioning signals and a JMJC domain, indicating that it might have a novel function in the nucleus. ${ }^{18-22}$ Recent studies indicate that JMJD6 is present in various organisms ${ }^{20}$ and is mainly located in the nucleus. ${ }^{16,21-25}$ Its protein sequence contains JMJC folds, ${ }^{26,27}$ which are doublestranded $\beta$-helix or typical cupin folds shared by all 2-oxopentane acid-dependent dioxygenases (2-OG), ${ }^{28,29}$ and catalytic studies also identified JMJD6 as a functional 2-OG oxygenase. ${ }^{30,31}$
Elevated levels of some tricarboxylic acid cycle intermediates can inhibit 2-OG oxygenases in some tumors. ${ }^{32,33}$ The $\mathrm{Fe}^{2+}$ binding site of the catalytic center of JMJD6 is located at the opening of the double-stranded beta-helix fold, forming a barrel structure, which is important for the enzymatic activity of JMJD6. ${ }^{26,27,34,35}$ The demethylation of dimethylated arginine residues was shown to be catalyzed by JMJD6 on histone $\mathrm{H} 3$ arginine 2 (H3R2me2) and histone $\mathrm{H} 4$ arginine 3 (H4R3me2). ${ }^{27,34}$ However, the current consensus is that JMJD6 is a lysyl 5hydroxylase, not a demethylase. ${ }^{10,26,36}$ Studies also demonstrated that JMJD6 interacts with bromodomain-containing protein 4 (Brd4), suggesting that JMJD6 and Brd4 are joined together on anti-pause enhancers (A-PEs). ${ }^{34}$ The ablation of the JMJD6 gene in Caenorhabditis elegans delayed the engulfment of apoptotic cells, ${ }^{15,37}$ and depletion of JMJD6 promoted apoptosis, induced cell death by sensitizing cells to DNA damaging reagent, and repressed cell tumorigenesis and proliferation, which is mediated by $553 .^{9}$ Furthermore, some studies have suggested that the high expression of JMJD6 is related to tumor growth, tumor metastasis and high tumor pathological classification. ${ }^{9,38-41}$

Recent studies indicate that JMJD6 may play a role in other reactions, such as inflammation, infections and immune responses. These reports showed a decrease in the expression of JMJD6 in T lymphocytes of patients who had chronic hepatitis B virus infection, ${ }^{42}$ and the proliferation of CD4+ T cells could be

\footnotetext{
'Department of Gynecology and Obstetrics, Key Laboratory of Obstetric and Gynecologic and Pediatric Diseases and Birth Defects of the Ministry of Education, West China

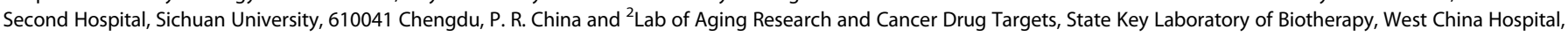
Sichuan University and Collaborative Innovation Center, No. 17, Block 3, Southern Renmin Road, 610041 Chengdu, Sichuan, P. R. China

Correspondence: Xiawei Wei (xiaweiwei@scu.edu.cn)

These authors contributed equally: Heng Zheng, Yan Tie
}

Received: 19 February 2019 Revised: 16 May 2019 Accepted: 20 May 2019

Published online: 26 July 2019 
inhibited by reducing the expression of JMJD6 in normal peripheral blood mononuclear cells. ${ }^{42,43}$ For the expression of JMJD6 in immune cells and its role in RNA metabolism, replication of viral RNA may be necessary. ${ }^{44,45}$

Recent studies also indicate that expression of JMJD6 in some human cancers, such as lung adenocarcinoma, ${ }^{39}$ breast ductal carcinomas, ${ }^{40}$ and colon adenocarcinomas, ${ }^{9}$ is evidently upregulated. These data indicate that JMJD6 may be an attractive therapeutic target for cancer intervention. However, the expression of JMJD6 in ovarian cancer has not been reported. Additionally, no JMJD6 inhibitors have been developed and applied to targeted cancer research. In our research, the expression of JMJD6 was detected by tissue microarray immunohistochemical staining, and we found high expression of JMJD6 in ovarian cancer, which was correlated with poor prognosis. Furthermore, we designed inhibitors based on the crystal structure of the jmjC domain of JMJD6 and selected the most active inhibitor, SKLB325, and conducted a study of the antitumor effects of SKLB325 on ovarian cancer in vivo and in vitro.

\section{METHODS}

Human ovarian tissue samples

JMJD6 expression in 151 patients with ovarian masses who underwent surgical excision between February 2009 and February 2013 was analyzed, including two borderline ovarian tumor tissues, three para-carcinoma tissues, 14 ovarian cancer metastasis tissues, and 132 primary ovarian cancer tissues (Scheme 1). None of these patients received preoperative radiotherapy or chemotherapy. All patients were followed up for at least 5 years after the operation. Pathological diagnosis and staging of tumors was in accordance with $\mathrm{WHO}$ and FIGO criteria. ${ }^{2}$ Sections were incubated with anti-JMJD6 monoclonal mouse antibody (Santa Cruz Biotechnology, sc-28348, 1:50). Then, slices were incubated with biotinylated secondary antibodies and a third antibody. Diaminobenzidine was used for detecting colorimetric activity. Negative controls were included by omitting the primary antibody. Five random fields of view at $\times 200$ were examined for each tissue. Referring to the previous literature for the classification of JMJD6 expression, ${ }^{41}$ our staining results were scored as follows: low staining $(1+),<10 \%$ of tumor cells positively stained; intermediate staining $(2+), 10-80 \%$ of tumor cells with positive staining; strong staining $(3+)$, positive staining in $>80 \%$ of tumor cells. The samples with $1+$ and $2+$ staining were defined as the "low expression group," and those with $3+$ staining were classified as the "high expression group."

\section{Chemistry}

All materials and reagents used in our study were purchased from commercial vendors without further purification. A Bruker AV-400 spectrometer was used to record ${ }^{13} \mathrm{C}$ NMR and ${ }^{1} \mathrm{H}$ NMR spectra at 400 and $100 \mathrm{MHz}$, respectively. Thin layer chromatography (TLC) was used for monitoring reactions, and it was performed on Merck silica gel 60 F-254 thin layer plates. High-performance liquid chromatography (HPLC) was used for determining final compounds whose purities were all $>95 \%$. All the determinations were performed on a Waters 2695 HPLC system equipped with a Kromasil C18 column $(4.6 \mathrm{~mm} \times 250 \mathrm{~mm}, 5 \mu \mathrm{m})$.

Ethyl acetoacetate $(10 \mathrm{~g}, 77 \mathrm{mmol})$ and 6-methyl-2-(methylthio) pyrimidin-4-ol (2): sodium carbonate $(16.29 \mathrm{~g}, 154 \mathrm{mmol})$ were added to a solution of 2-methyl-2-thiopseudourea sulfate $(15.91 \mathrm{~g}$, $85 \mathrm{mmol})$ in water $(200 \mathrm{~mL})$ at room temperature and then churned overnight. After neutralizing to $\mathrm{pH}=8$, filtrating, and drying under vacuum overnight, the solid was collected. The product was used without further purification.

For 2-hydrazinyl-6-methylpyrimidin-4-ol (3): To a suspension of 6-methyl-2-(methylthio) pyrimidin-4-ol $(2.1 \mathrm{~g}, 13 \mathrm{mmol})$ and potassium carbonate $(140 \mathrm{mg}, 1 \mathrm{mmol})$ in 2-propanol $(40 \mathrm{~mL})$, hydrazine hydrate $(6.3 \mathrm{~mL}, 130 \mathrm{mmol})$ was added dropwise at room temperature. After cooling to room temperature, the precipitate was filtered, washed with methanol, and dried under vacuum overnight. The product was used without further purification.

For 2-(2-(2-hydroxybenzylidene) hydrazinyl)-6-methylpyrimidin4-ol (SKLB325): A suspension of 2-hydrazinyl-6-methylpyrimidin-4ol $(1.8 \mathrm{~g}, 13 \mathrm{mmol})$ and 2-hydroxybenzaldehyde $(3.14 \mathrm{~g}, 26 \mathrm{mmol})$ in methanol $(30 \mathrm{~mL})$ was heated at reflux overnight. ${ }^{1} \mathrm{H}$ NMR $\left(400 \mathrm{MHz}, \mathrm{DMSO}-d_{6}\right) \delta 11.26(\mathrm{br} \mathrm{s}, 2 \mathrm{H}), 9.98(\mathrm{br} \mathrm{s}, 1 \mathrm{H}), 8.36(\mathrm{~s}, 1 \mathrm{H})$, $8.04(\mathrm{~d}, J=7.8 \mathrm{~Hz}, 1 \mathrm{H}), 7.29-7.14(\mathrm{~m}, 1 \mathrm{H}), 6.89-6.82(\mathrm{~m}, 2 \mathrm{H}), 5.52$ $(\mathrm{s}, 1 \mathrm{H}), 2.08(\mathrm{~s}, 3 \mathrm{H}) .{ }^{13} \mathrm{C}$ NMR $\left(101 \mathrm{MHz}, \mathrm{DMSO}-d_{6}\right) \delta 163.15$, $156.54,152.82$, 143.04, 131.26, 128.42, 120.79, 119.66, 116.46, 101.53, 23.08. ESI-ms (m/z):245.1[M+H] ${ }^{+}$.

\section{SPR analysis}

Biacore T200 instruments were used for the surface plasmon resonance (SPR) procedure at $25^{\circ} \mathrm{C}$. BIA evaluation software (GE Healthcare) was used to determine the $K_{\mathrm{D}}$ value of the compounds.

\section{Drug concentration}

The inhibitor (SKLB325) powder was completely dissolved in DMSO at a concentration of $40000 \mu \mathrm{M}$.

Cell lines and cell culture

The cell lines SKOV3, ES2, CP70, and A2780s used in this study were purchased from the American Type Culture Collection (ATCC, Manassas, VA) and were cultured in Dulbecco's modified Eagle's medium (DMEM) supplemented with $10 \%$ heat-inactivated fetal bovine serum (FBS), $100 \mathrm{U} / \mathrm{ml}$ penicillin, $0.1 \mathrm{mg} / \mathrm{ml}$ streptomycin and $2 \mathrm{mmol}$ L-glutamine. DMEM and FBS were purchased from Gibco. Human umbilical vein endothelial cells (HUVECs) were supported by the Department of Obstetrics \& Gynecology, West China Second University Hospital, Sichuan University, Chengdu, China and were isolated from human umbilical cord. Cells used in our study were incubated at $37^{\circ} \mathrm{C}$ in a humidified circumstance containing $5 \% \mathrm{CO}_{2}$. Trypsin was used for passaging cells at a ratio of $1: 3$ every 3 days.

\section{CCK-8 cell proliferation assay}

SKOV3 cells were seeded in 96-well plates at 3000-5000 cells/well in $100 \mu \mathrm{l}$ of medium and cultured for 24,48 , or $72 \mathrm{~h}$. After incubation periods, $100 \mu$ l of DMEM containing $10 \%$ Cell Counting Kit-8 (CCK-8) (Beyotime, China) was added to each well. To form water-insoluble formazan, cells were incubated for another $3 \mathrm{~h}$ at

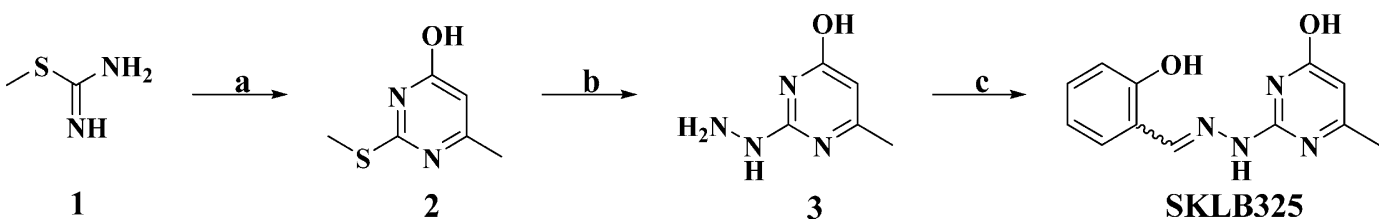

Scheme 1 The synthetic route for SKLB325. Reagents and conditions: a ethyl acetoacetate, $\mathrm{Na}_{2} \mathrm{CO}_{3}, \mathrm{H}_{2} \mathrm{O}$, rt, overnight; $\mathbf{b}$ hydrazine hydrate, $\mathrm{K}_{2} \mathrm{CO}_{3}$, isopropanol, reflux, overnight; c 2-hydroxybenzaldehyde, methanol, reflux, overnight 
$37^{\circ} \mathrm{C}$. A microplate reader was used to measure the absorbance at $450 \mathrm{~nm}$ according to the instructions. Each concentration was replicated in at least four wells. Vehicle, control, and blank groups were created simultaneously: the vehicle group contained cells with DMSO concentrations equal to that of corresponding drug wells and $10 \%$ CCK-8; the control group contained cells and $10 \%$ CCK-8; the blank group contained only 10\% CCK-8. Data for inhibition were calculated by the following formula: Inhibition $=$ (SKLB325- vehicle)/(control-blank), viability = 1-inhibition.

\section{Confocal microscopy}

The slides of SKOV3 cells were imaged after immunofluorescence staining, which was performed with JMJD6 (Abcam, 1:50) and p53 (Santa Cruz, 1:50) primary antibodies. Alexa Fluor 488-conjugated goat-anti-rabbit antibody (Invitrogen, 1:100) and Alexa Fluor 594conjugated goat-anti-rat antibody (Invitrogen, 1:100) were used as the secondary antibodies. Then, the cells were stained with $5 \mu \mathrm{g} /$ $\mathrm{ml}$ DAPI for $10 \mathrm{~min}$, followed by washing and imaging using a Leica TCS SP5 confocal microscope. SKOV3 cells stained with secondary antibodies but without primary antibodies were used as the negative controls.

Matrigel endothelial tube formation assay

Matrigel (BD, USA) was melted at $4{ }^{\circ} \mathrm{C}$ overnight, and $100 \mu \mathrm{l}$ of Matrigel/well was vertically plated into 24-well plates and cultured at $37^{\circ} \mathrm{C}$ for $45 \mathrm{~min}$. Then, $1 \times 10^{5}$ HUVECs and $4 \mu \mathrm{M}$ of SKLB325 were added into each well simultaneously with cultured Matrigel. DMSO in media was used as a control. After culturing at $37^{\circ} \mathrm{C}$ for $4 \mathrm{~h}$, a phase contrast microscope (OLYMPUS inverted digital camera) was used to observe the tube formation, and the number of tubes was counted manually in five random microscopic fields at $\times 100$ magnification.

\section{Transwell migration assay}

A total of $4 \times 10^{4}$ HUVECs, SKOV3, A2780s and ES2 cells were resuspended in $200 \mu \mathrm{L}$ DMEM medium with $4 \mu \mathrm{M}$ of the inhibitor and placed in the upper chamber. DMSO in media was used as a control. Then, the chamber was placed in a 24-well plate containing $600 \mu \mathrm{L}$ DMEM and $10 \%$ FBS. After incubation at $37^{\circ} \mathrm{C}$ overnight, migrating cells were counted manually in five random microscopic fields at $\times 200$ magnification.

Quantitative real-time reverse transcription PCR

An RNA Simple Total RNA Kit (Tiangen Biotech, China) was used for preparing total cellular RNA, and a PrimeScript RT reagent Kit with gDNA Eraser (TaKaRa) was used for reverse transcribing isolated RNA into cDNA. Sso Advanced SYBR Green Supermix (BioRad) was used for quantifying all gene transcripts by qPCR with a two-step PCR procedure. A CFX96 Real-Time System (Bio-Rad) was used to measure mRNA levels. Expression of GAPDH served as the internal control. Sense and antisense primers were as follows: $5^{\prime}$ TITAACTCTGGTAAAGTGGA-3' and 5'-GAATCATATTGGAACATGTA$3^{\prime}$ for GAPDH; 5'-GACGACCTCAACGCACAGTA-3' and 5'-CACC TAATTGGGCTCCATCT-3' for PUMA; 5'-GGACCTGGAGACTCTCA-3' and $5^{\prime}$-CCTCTTGGAGAAGATCA-3' for p21; 5'-GTTCCGAGAGCTGA ATGAGG-3' and $5^{\prime}$-TCTGAGTCAGGCCCTTCTGT-3' for p53.

\section{Apoptosis assay}

Aliquots of $5 \times 10^{4}$ cells were plated in six-well plates in triplicate. After $4 \mathrm{~h}$, we changed the medium to $2 \mathrm{ml}$ DMEM containing $10 \%$ fetal bovine serum and a series of drug concentrations as well as corresponding doses of DMSO. Cells were incubated for $72 \mathrm{~h}$ to $80 \%$ confluence, at which time both the attached cells and floating cells were trypsinized, collected in flow cytometry tubes and washed twice with cold PBS. Cells were resuspended in $100 \mu \mathrm{l}$ of $1 \mathrm{x}$ binding buffer and stained with FITC-Annexin V and PI (BD Pharmingen, $\mathrm{CA}$ ) for $10 \mathrm{~min}$ in the dark and then analyzed by flow cytometry (Novoexpress, China).
Western blot analysis

Samples from each group were lysed in modified RIPA lysis buffer (Beyotime Biotech, China), which contained protease inhibitor cocktail (Sigma), and then placed on ice for $30 \mathrm{~min}$. Then, samples were centrifuged at $13000 \mathrm{rpm}$ and $4{ }^{\circ} \mathrm{C}$ for $30 \mathrm{~min}$. Protein concentrations were determined using a BCA protein assay kit (Pierce, Thermo Fisher Scientific, Inc., Waltham, MA, USA). Equal quantities of protein were loaded onto $12.5 \%$ SDS-PAGE for electrophoresis, followed by transfer to PVDF membranes (Millipore). After blocking with $5 \%$ nonfat milk for $2 \mathrm{~h}$, membranes were immunoblotted with rabbit anti-human polyclonal antibodies against p53 (1:5000, Santa Cruz), p21 (1:1000, CST) and PUMA (1:1000, Abcam) overnight at $4{ }^{\circ} \mathrm{C}$. Then, appropriate secondary antibodies (goat-anti-rabbit antibody, 1:10000; ZSGB-BIO, Beijing, China) were used for incubating the blots for $1 \mathrm{~h}$ at $37^{\circ} \mathrm{C}$. GAPDH was used as a control (1:5000; Zen-BioScience, Chengdu, China). A western blotting luminol reagent (Millipore, MA, USA) was used to visualize the immunoreactive bands.

Establishment of a peritoneal carcinomatosis model in BALB/C nude mice

Female athymic BALB/C nude mice used in this study were purchased from HFK Bioscience (Beijing, China) at 5-6-weeks-old. The mice were housed in microisolator cages and fed sterilized water and food.

Five nude mice were used for subcutaneous (s.c.) injection with $5 \times 10^{6}$ SKOV3 cell suspensions in their right backs. When the tumor diameter reached approximately $1 \mathrm{~cm}$, tumors were minced into small particles within a diameter of $1 \mathrm{~mm}$ after being isolated free of normal and necrotic tissues. DMEM medium was used to resuspend these tumor tissues in a total volume of $10 \mathrm{ml}$. Then, $0.5 \mathrm{ml}$ of the resuspension was inoculated i.p. in the right lower quadrant of fifteen nude mice with a 14-gauge needle. As in previous studies performed in our laboratory, as early as 7 days after i.p. inoculation, tumor nodules could be observed, and extensive dissemination of intraperitoneal carcinomatosis was also detected. For this, 7 days after injection of tumor particles, therapeutic studies were started.

\section{Therapy studies}

First, the drug was prepared (mixture of $20 \mu$ of SKLB325 ultimate solution and $80 \mu \mathrm{l}$ of normal saline (NS)). As a control for our drug, equal concentrations of DMSO were used in the vehicle group.

The mice mentioned above were randomly assigned into three groups ( $n=5)$ : (1) untreated mice, (2) mice treated with $100 \mu \mathrm{l}$ of NS and DMSO, or (3) mice treated with $100 \mu$ of NS and SKLB325 $(10 \mathrm{mg} / \mathrm{kg})$. Mice received i.p. injections every three days for eight doses total and were monitored for abdominal distension, tumor burden and other pathologies before i.p. administration. Three days after the last administration, all fifteen mice were sacrificed, and tumors were subsequently isolated, counted and weighed. We also used five nude mice without nodules as the normal control group from the same batch as the mice in the three experimental groups.

To detect the effect of our compound against ovarian cancer, we observed the survival time as previously described. When mice were at dying status, they were sacrificed, and we recorded the day when they were sacrificed as their survival time.

\section{Immunohistochemistry}

After fixing the intraperitoneal tumors in 4\% paraformaldehyde all night, we embedded them in paraffin and cut the wax blocks into 3-4 mm slices. For antigen retrieval, slices were dewaxed first, after which the slices were immersed in $10 \mathrm{mmol} / \mathrm{L}$ sodium citrate buffer ( $\mathrm{pH} 6.0$ ), followed by heating in an autoclave that was in saturated conditions for $3 \mathrm{~min}$. Then, $3 \% \mathrm{H}_{2} \mathrm{O}_{2}$ was used for quenching the endogenous peroxidase activity, the procedure was performed in a dark room at room temperature for $10 \mathrm{~min}$, 
and normal goat serum was used for blocking the nonspecific binding at $37^{\circ} \mathrm{C}$ for $20 \mathrm{~min}$. Next, the slices were incubated with primary antibodies against P53, PUMA, and Ki-67 at $4{ }^{\circ} \mathrm{C}$ overnight. Then, slices were incubated with biotinylated secondary antibodies at $37^{\circ} \mathrm{C}$ for 40 min; subsequently, they were incubated with a third antibody at $37^{\circ} \mathrm{C}$ for $40 \mathrm{~min}$. Diaminobenzidine was used for the colorimetric detection. Sections of the intraperitoneal tumours were used to detect vessel density with anti-VEGF and anti-CD31 antibodies, of which CD31 antibody was used for frozen sections. Negative controls were included by omitting primary antibody.

\section{In situ TUNEL}

Apoptosis in human ovarian cancer xenograft tumours was examined using a TUNEL kit (Merck Millipore) following the manufacturer's instructions. Five tumours per group were analyzed. Fluorescence microscopy was used to quantify the number of TUNEL-positive cells, and the apoptotic index in at least five random fields per group at $\times 400$ magnification was calculated.

Toxicity evaluation

Diarrhea, weight loss, ruffling of fur, anorexia, behavioral changes, and even deaths as relevant indexes of another 15 mice were observed continuously to detect potential toxicity to mice in response to treatment in our study. In addition, sections of heart, lung, liver, spleen, and kidney tissues were stained with hematoxylin and eosin (H\&E) and histologically evaluated by professional pathologists. In addition, routine analysis and biochemical analysis of blood was performed for every mouse.

\section{Statistical analysis}

The relationship between JMJD6 expression and clinicopathological characteristics was analyzed by Pearson's $X^{2}$ test. Overall survival (OS), defined as time from operation to death or date of last follow-up, disease-free survival (DFS), defined as time from operation to relapse, and death or date of last follow-up were studied. Survival curves were analyzed by the Kaplan-Meier method, and the log-rank test was used for comparing groups. Statistical analysis was performed using IBM SPSS Statistics 23. A $P$ value $<0.05$ was considered to be statistically significant.

\section{RESULTS}

The expression of JMJD6 is correlated with some clinicopathologic characteristics

The expression of JMJD6 protein in 146 ovarian cancer tissue samples was detected by immunohistochemical staining to explore the relationship between the expression of JMJD6 and the clinicopathological characteristics of ovarian cancer patients. As shown in Fig. 1a and Table 1, JMJD6 was highly and lowly expressed in $90(61.64 \%)$ and $56(38.36 \%)$ of the 146 ovarian

A
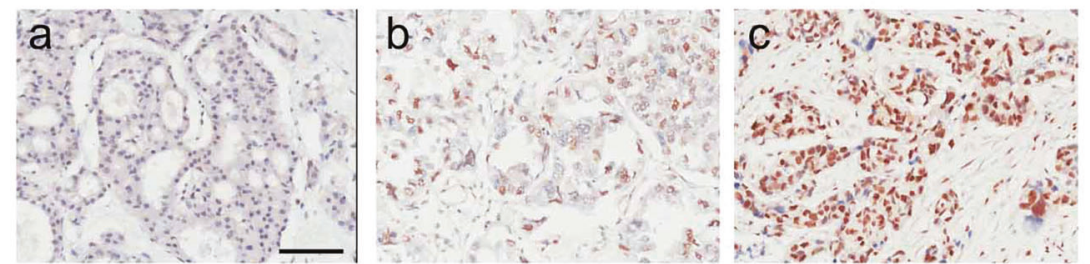

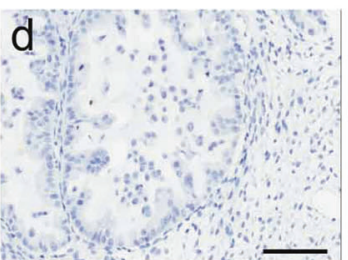

B

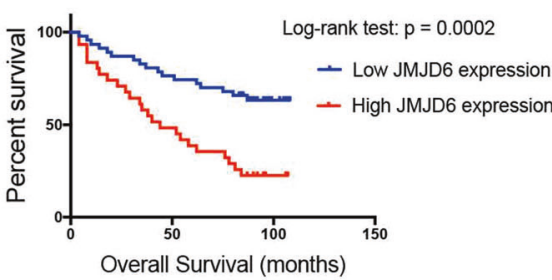

$\mathrm{D}$

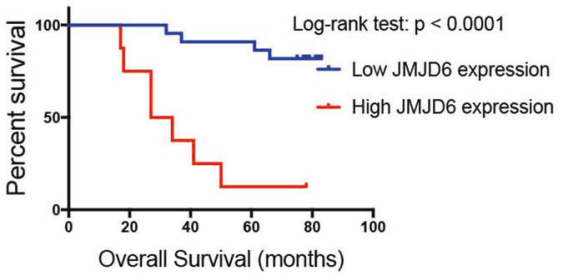

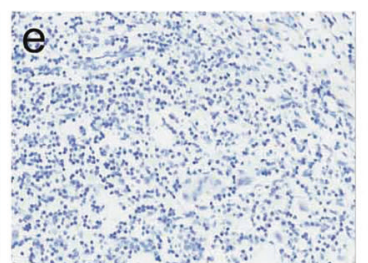

C

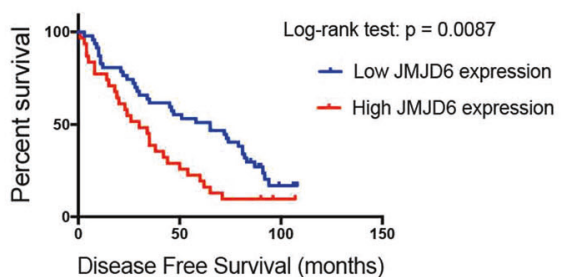

E

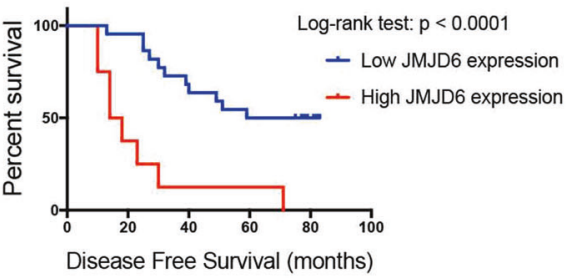

Fig. 1 Kaplan-Meier estimates of OS and DFS according to JMJD6 expression groups. a JMJD6 protein expression in ovarian cancer tissues detected by immunohistochemistry analysis. a Low staining, b Intermediate staining, c Strong staining of JMJD6 protein, d Negative control (normal adjacent tissues of ovarian cancer), e Primary antibody-free negative control, magnification, $\times 200$. b Overall survival of serous ovarian cancer, log-rank test: $p=0.0002$. c Disease-free survival of serous ovarian cancer, log-rank test: $p=0.0087$. d Overall survival of mucinous ovarian cancer, log-rank test: $p<0.0001$. e Disease-free survival of mucinous ovarian cancer, log-rank test: $p<0.0001$ 


\begin{tabular}{|c|c|c|c|c|}
\hline \multirow[t]{2}{*}{ Variables } & \multirow[t]{2}{*}{ No. } & \multicolumn{2}{|c|}{$\begin{array}{l}\text { JMJD6 protein } \\
\text { expression }\end{array}$} & \multirow[t]{2}{*}{$P$ value } \\
\hline & & Low & High & \\
\hline Age & 146 & 90 & 56 & 0.026 \\
\hline$<60$ & 111 & 74 & 37 & \\
\hline$\geq 60$ & 35 & 16 & 19 & \\
\hline Tumor diameter & 146 & 90 & 56 & 0.140 \\
\hline$<10 \mathrm{~cm}$ & 58 & 40 & 18 & \\
\hline$\geq 10 \mathrm{~cm}$ & 88 & 50 & 38 & \\
\hline Pathological grade & 118 & 69 & 51 & 0.136 \\
\hline 1 & 13 & 10 & 3 & \\
\hline $2-3$ & 105 & 58 & 47 & \\
\hline Clinical stage & 146 & 90 & 56 & $<0.001$ \\
\hline $1-I I$ & 45 & 38 & 7 & \\
\hline III-IV & 101 & 52 & 49 & \\
\hline pT status & 146 & 90 & 56 & 0.015 \\
\hline pT 1 & 9 & 9 & 0 & \\
\hline pT 2-3 & 137 & 81 & 56 & \\
\hline pN status & 146 & 90 & 56 & 0.003 \\
\hline $\mathrm{pN} 0$ & 106 & 73 & 33 & \\
\hline pN 1 & 40 & 17 & 23 & \\
\hline pM status & 146 & 90 & 56 & 0.765 \\
\hline pM 0 & 114 & 71 & 43 & \\
\hline pM 1 & 32 & 19 & 13 & \\
\hline Relapse & 146 & 90 & 56 & 0.002 \\
\hline Yes & 116 & 64 & 52 & \\
\hline No & 30 & 26 & 4 & \\
\hline
\end{tabular}

cancer patients, respectively, according to the intensity of immunoreaction. As shown in Table 1, high expression of JMJD6 was significantly associated with age $(p=0.026)$, clinical stage $(p<0.001)$, pT status $(p=0.015), \mathrm{pN}$ status $(p=0.003)$ and relapse $(p=0.002)$. However, no significant relationship was found between JMJD6 protein expression and characteristics such as tumor diameter $(p=0.140)$, pathological grade $(p=0.136)$ and $\mathrm{pM}$ status $(p=0.765)$.

JMJD6 is a marker of poor prognosis in ovarian cancer

We next investigated the prognosis of JMJD6 expression in ovarian cancer. A cohort of 146 ovarian cancer samples was detected by immunohistochemical staining. We found that the nuclear staining of JMJD6 varied from low levels (Fig. 1a, a) to intermediate (b) and strong levels (c) in ovarian cancer tissue. In addition, normal adjacent tissues of ovarian cancers were used for the negative controls (Fig. 1a, d), and primary antibody-free staining of ovarian cancer tissues was used for the primary antibody-free negative controls (Fig. 1a, e). JMJD6 was highly expressed in 31 (39.74\%) of the 78 serous ovarian cancer patients and in eight (26.67\%) of the 30 mucinous ovarian cancer patients. Kaplan-Meier survival analysis indicated that serous and mucinous ovarian cancer patients with high JMJD6 expression had a significantly shorter median overall survival as well as diseasefree survival $(p=0.0002, p=0.0087, p<0.0001$, and $p<0.0001$, respectively, Fig. $1 \mathrm{~b}-\mathrm{e})$. Kaplan-Meier survival analysis also showed that patients with high JMJD6 expression had a significantly shorter median overall survival and disease-free survival $(p<0.0001$ and $p=0.0001)$.

As shown in Tables 2 and 3, in terms of patient outcome, univariate Cox regression analysis indicated that JMJD6 expression $(p<0.001$ and $p<0.001)$, age $(p=0.003$ and $p=0.001)$, pT status $(p<0.001$ and $p<0.001)$, $\mathrm{pN}$ status $(\mathrm{p}<0.001$ and $p<0.001)$, and pM status $(p<0.001$ and $p<0.001)$ were significantly associated with OS as well as DFS. Furthermore, multivariate Cox regression

\begin{tabular}{|c|c|c|c|c|c|c|}
\hline \multirow[t]{2}{*}{ Variables } & \multicolumn{3}{|c|}{ Univariate analysis } & \multicolumn{3}{|c|}{ Multivariate analysis } \\
\hline & RR & $95 \% \mathrm{Cl}$ & $P$ value & RR & $95 \% \mathrm{Cl}$ & $P$ value \\
\hline JMJD6: \% of stained cells & 2.955 & $1.879-4.646$ & $<0.001$ & 2.005 & $1.169-3.438$ & 0.012 \\
\hline Age & 1.031 & $1.011-1.051$ & 0.003 & 1.009 & $0.987-1.031$ & 0.411 \\
\hline pT status & 0.098 & $0.036-0.269$ & $<0.001$ & 0.131 & $0.036-0.482$ & 0.002 \\
\hline $\mathrm{pN}$ status & 5.807 & $3.656-9.226$ & $<0.001$ & 1.113 & $0.559-2.216$ & 0.76 \\
\hline pM status & 6.226 & $3.823-10.139$ & $<0.001$ & 2.935 & $1.427-6.04$ & 0.003 \\
\hline Tumor diameter & 0.778 & $0.354-1.711$ & 0.533 & 0.692 & $0.307-1.558$ & 0.373 \\
\hline Pathological grade & 0.376 & $0.092-1.535$ & 0.173 & 3.17 & $0.623-16.141$ & 0.165 \\
\hline
\end{tabular}

$R R$ relative risk, $95 \% \mathrm{Cl} 95 \%$ confidence interval

Table 3. Univariate and multivariate analysis showing the DFS rate for ovarian cancer patients

\begin{tabular}{|c|c|c|c|c|c|c|}
\hline JMJD6: \% of stained cells & 2.783 & $1.769-4.377$ & $<0.001$ & 1.385 & $0.812-2.364$ & 0.232 \\
\hline Age & 1.032 & $1.012-1.052$ & 0.001 & 1.008 & $0.988-1.029$ & 0.433 \\
\hline pT status & 0.09 & $0.033-0.246$ & $<0.001$ & 0.107 & $0.027-0.425$ & 0.001 \\
\hline pN status & 5.698 & $3.587-9.053$ & $<0.001$ & 1.178 & $0.605-2.295$ & 0.63 \\
\hline Pathological grade & 0.352 & $0.086-1.44$ & 0.146 & 2.876 & $0.519-15.923$ & 0.226 \\
\hline
\end{tabular}


6
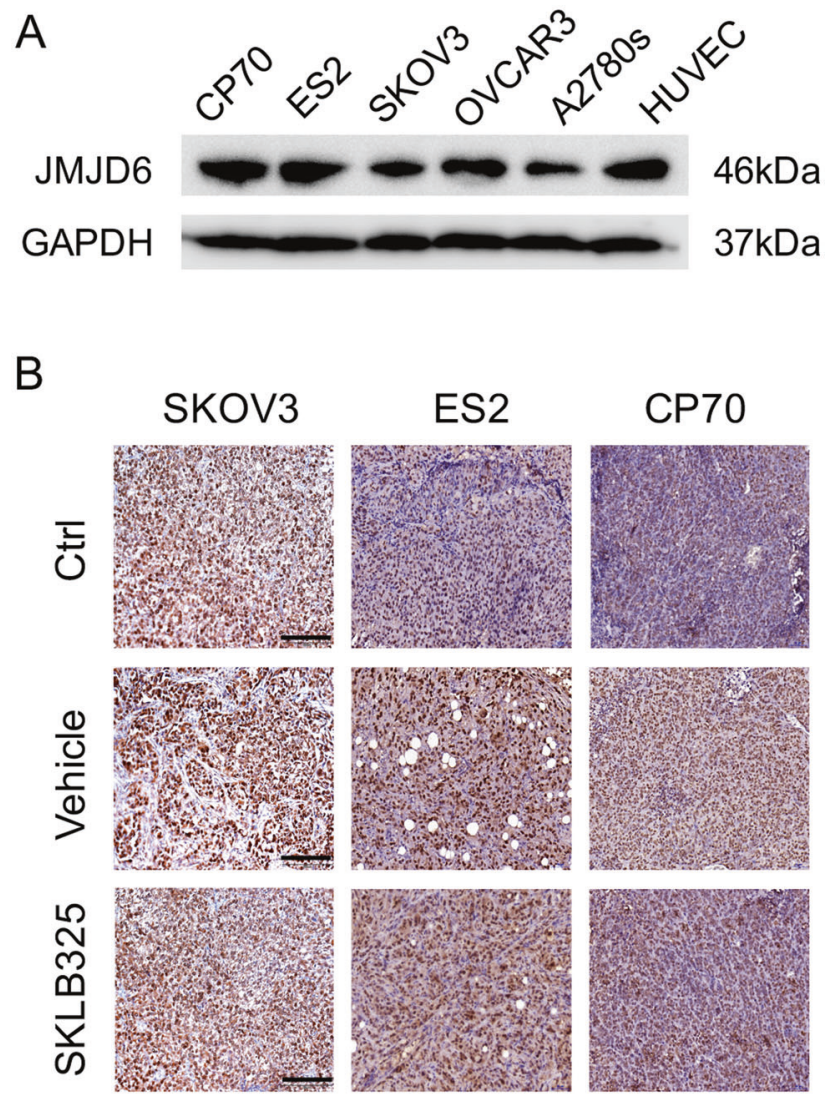

Fig. 2 Expression of JMJD6 in vitro and in vivo. a JMJD6 is highly expressed in ovarian cancer cells and HUVECs, which was examined by western blot. b JMJD6 is highly expressed in tumor tissues isolated from an intraperitoneal dissemination xenograft mouse model

analysis was used to determine whether the expression of JMJD6 could be an independent predictor for OS or DFS of ovarian cancer. The results revealed that expression of JMJD6 $(p=0.012)$, pT $(p=0.002)$ status and $\mathrm{pM}(p=0.003)$ status remained independent prognostic factors for OS, although no significant association was found for JMJD6 as an independent prognostic factor for DFS ( $p=0.232$ ) (Tables 2 and 3). Altogether, these data showed that expression of JMJD6 may play a role in predicting OS in ovarian cancer.

Virtual screening to retrieve new potential inhibitors for JMJD6 As JMJD6 was highly expressed in vitro and in vivo, as well as in HUVECs, which was examined by western blot and immunohistochemistry (Fig. 2a, b), we designed inhibitors of this molecule. To identify new inhibitors of JMJD6, we first screened compounds from several libraries according to a previous approach. ${ }^{46}$ The crystal structure of the jmjC domain of JMJD6 (PDB entry: 3LDB) was used to prepare the receptor structure for molecular docking. The original ligand was large enough to cover all the catalytic site, and the sphere containing the residues that stayed within $8 \AA$ represented the active site; here, the active site is actually the binding site of a-ketoglutaric acid (a-OG). The Charmm force field was assigned. The GOLD program was used for molecular docking, and Goldscore was chosen to rank the compounds. We finally selected 20 agents from the top ranked compounds to carry out further experimental validation. One compound, namely, 2-(2-(2hydroxybenzylidene) hydrazinyl)-6-methylpyrimidin-4-ol (termed SKLB325, Fig. 3a), was the most active one and showed a binding affinity $\left(K_{D}\right)$ value of $0.755 \mu \mathrm{M}$ (Fig. $3 \mathrm{~b}$ ), and the $\mathrm{IC}_{50}$ value of the biochemical potency of the inhibitor SKLB325 was $0.7797 \mu \mathrm{M}$ (Supplementary Fig. 1).

Predicted binding mode of SKLB325 with JMJD6

The binding mode of SKLB325 in the active pocket of JMJD6 was predicted by molecular docking. Figure $3 \mathrm{C}$ shows a possible binding mode of SKLB325 with JMJD6. SKLB325 is suitably located in the binding site of a-OG. A strong coordination interaction is formed between the pyrimidine nitrogen (3-N) and $\mathrm{Fe}(\mathrm{II})$. In addition, the 2-position amino group forms a hydrogen bond with Asp189.

JMJD6 inhibition suppresses proliferation, induces apoptosis in ovarian cancer cells, inhibits angiogenesis in HUVECs, and inhibits migration in ovarian cancer cells and HUVECs in vitro

To explore the inhibitory effects on in vitro proliferation of ovarian cancer cells, CCK-8 was used to investigate the proliferation of ovarian cancer cells. As shown in Fig. 4a, with increasing SKLB325 concentration, the inhibitory effect also increased, exhibiting a significant dose-response relationship. There was a significant difference between the drug group across different doses and the control group $(p<0.05)$. These results indicated that SKLB325 had significant inhibitory effects on the in vitro proliferation of ovarian cancer cells. Furthermore, the most effective concentration at which JMJD6 inhibited SKOV3 cell growth was $4 \mu \mathrm{M}$, and the optimal duration of action was $72 \mathrm{~h}$.

We next detected whether SKLB325 affected cell apoptosis. SKOV3, ES2 and A2780s cells treated with various concentrations were analyzed by PI and annexin V double staining. The same dose of DMSO according to the concentration of $4 \mu \mathrm{M}$ was used in the control group. The results indicated that apoptotic cells observably increased $(35.27 \pm 1.56 \%, 28.39 \pm 1.19 \%)$ in response to $4 \mu \mathrm{M}$ treatment compared with those in the control group (3.57 \pm $0.74 \%, 4.32 \pm 1.09 \%$, Fig. $4 \mathrm{~b}$ and Supplementary Fig. 2A). Additionally, with increasing SKLB325 concentration, the percentage of apoptotic cells increased, suggesting that JMJD6 inhibition significantly promoted apoptosis in a dose-dependent manner. There were no apparent differences between various concentrations of SKLB325 in the levels of necrotic cells. However, the percentage of dead cells (sum of apoptotic cells and necrotic cells) increased $(36.63 \pm 1.22 \%$ versus $4.52 \pm 0.60 \%, 32.17 \pm 1.33 \%$ versus $3.78 \pm 0.48 \%, 21.00 \pm 0.01 \%$ versus $7.79 \pm 1.08 \%,{ }^{*} p<0.05,{ }^{* *} p<$ $0.01,{ }^{* * *} p<0.001$, Fig. 4 C and Supplementary Fig. $2 \mathrm{~A}$ and 2B). The results indicated that SKLB325 significantly induced the death of ovarian cancer cells. We also detected whether SKLB325 affected apoptosis or death in HUVECs. As Supplementary Fig. 2C shows, there were no differences between various concentrations of SKLB325 in the levels of apoptotic and dead cells in HUVECs.

To investigate the effect of SKLB325 on in vitro angiogenesis, we focused on the functions of SKLB325 in endothelial cells (ECs). As shown in Fig. $4 d$, the quantification of capillary tubes showed a $65.09 \%\left(21.2 \pm 1.86 \%\right.$ versus $\left.7.4 \pm 0.6 \%,{ }^{* *} p<0.001\right)$ decrease at $4 \mathrm{~h}$ when HUVECs were seeded with $4 \mu \mathrm{M}$ of the inhibitor simultaneously. Furthermore, transwell migration assays were used to assess the migration capacity of HUVECs treated with SKLB325. As shown in Fig. 4e, the number of HUVECs was $67.00 \%$ $\left(119.4 \pm 8.68 \%\right.$ versus $\left.39.4 \pm 4.39 \%,{ }^{* * *} p<0.001\right)$ reduced following $4 \mu \mathrm{M}$ inhibitor treatment for $12 \mathrm{~h}$ in the transwell migration assay. Similarly, the numbers of SKOV3, A2780s and ES2 cells showed $66.91 \%\left(54.4 \pm 3.58 \%\right.$ versus $18.0 \pm 2.00 \%$, $\left.{ }^{* * *} p<0.001\right)$, $64.27 \% \quad\left(94.6 \pm 4.51 \%\right.$ versus $\left.33.8 \pm 3.03 \%,{ }^{* * *} p<0.001\right)$ and $74.12 \%\left(45.6 \pm 2.30 \%\right.$ versus $11.8 \pm 2.59 \%$, $\left.{ }^{* * *} p<0.001\right)$ reductions following $4 \mu \mathrm{M}$ inhibitor treatment for $12 \mathrm{~h}$ in the transwell migration assay. These observations suggested that SKLB325 inhibited capillary tube organization and disrupted preformed capillary tubes by HUVECs on Matrigel, as well as inhibited the migration of ECs and ovarian cancer cells. 
<smiles>Cc1cc(O)nc(N/N=C/c2ccccc2O)n1</smiles>

\section{SKLB325}

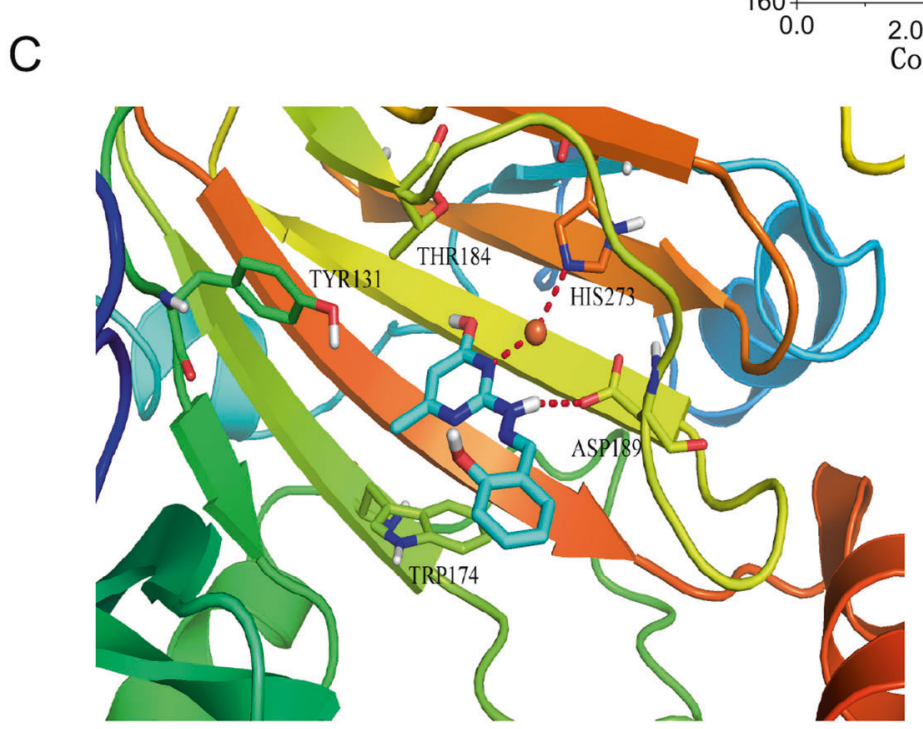

Fig. 3 Identification and characterization of the inhibitor SKLB325. a Chemical structure of the inhibitor SKLB325. b Binding affinity of SKLB325 with JMJD6 by SPR assays. The concentrations of SKLB325 injected over the biosensor chip surface immobilized with JMJD6 protein are indicated. The measurement yielded a KD of $0.755 \mu \mathrm{M}$. c Predicted binding mode of SKLB325 in the active pocket of JMJD6. SKLB325 is color coded with a carbon atom in cyan, a nitrogen atom in blue, and an oxygen atom in red. Fe(II) is in orange. Coordination and hydrogen bonds are shown in the red dashed line

SKLB325 upregulates the expression of p53 and its downstream effectors at both the mRNA and protein levels in vitro To explore the cellular functions of JMJD6, confocal microscopy was used to determine the subcellular localization of endogenous JMJD6 and p53 in SKOV3 cells. The results demonstrated that p53 protein colocalized with JMJD6 in the nucleus (Fig. 4f).

To evaluate the expression of p53 as well as its downstream effectors in response to treatment with SKLB325 in vitro, SKOV3 cells were collected $72 \mathrm{~h}$ after exposure to SKLB325 or the same dose of DMSO and analyzed by qRT-PCR and western blots. The results showed that compared with the control group, the expression of p53, p21, and PUMA in SKOV3 cells was increased by $130.24 \%, 237.15 \%$, and $841.36 \%$ in the SKLB325 group, respectively $\left({ }^{* * *} p<0.001\right)$. Similarly, the expression of $\mathrm{p} 53, \mathrm{p} 21$, and PUMA in ES2 cells was increased by $45.08 \%, 49.92 \%$, and $96.48 \%$ in the SKLB325 group $\left({ }^{* *} p<0.001\right)$, respectively. The expression of p53, p21, and PUMA in A2780s cells was increased by $265.10 \%, 613.73 \%$, and $401.70 \%$ in the SKLB325 group, respectively $\left({ }^{* *} p<0.001\right)$. Furthermore, the expression of $\mathrm{p} 53$, p21, and PUMA in HUVECs was increased by $54.96 \%, 20.04 \%$, $197.33 \%$ in the SKLB325 group ${ }^{* * *} p<0.001$ ) (Fig. 4 g), respectively. As shown in Fig. 4h, p53, p21, and PUMA protein levels were significantly upregulated after treatment with SKLB325 in SKOV3, ES2 and A2780s cells. As shown in Fig. 4i, VEGFR1 protein levels were significantly downregulated after treatment with SKLB325 in HUVECs. However, p53, p21, and PUMA protein levels were significantly upregulated in HUVECs. The results of the western blot analysis were consistent with the results observed by RT-PCR. In general, these results indicated that $\mathrm{p} 53$ and its downstream effectors were effectively upregulated at both the mRNA and protein levels after treatment with SKLB325 in vitro.

SKLB325 had antitumor activities in an intraperitoneal xenograft model

Then, we performed comparative analyses of JMJD6 and catalytic domain (JMJC) proteins of human and mouse; as shown in Supplementary Fig. 3A and B, mouse JMJD6 displays 98\% (403 aa) identity with human JMJD6 and mouse JMJC displays 99\% (165 aa) identity with human JMJC. The results demonstrated that it was reasonable to use the mouse model to explore the effects and side effects of SKLB325. As SKLB325 could induce apoptosis and inhibit cell proliferation, as shown above, this inhibitor had antitumor potential in vivo. Therefore, we established an intraperitoneal xenograft model to further evaluate the effect of SKLB325 in vivo. Tumor nodules in the two control groups were scattered on the kidney, pancreas, or parenchyma of the liver. In the SKLB325 group, the growth of intraperitoneal tumor nodules was limited to the pelvis, and organ and tissue invasion was absent. Tumor nodules varied in size, but they were more numerous and larger in the two control groups than in the SKLB325 group. The average weight of intraperitoneal tumor nodules was $1.56 \pm 0.70,1.04 \pm 0.62$, and $0.14 \pm 0.11 \mathrm{~g}$ in the control, vehicle and SKLB325 groups, respectively. Tumor weight was significantly reduced by 91 and $86 \%$ in the SKLB325 groups compared to the control and vehicle groups, respectively $(p<0.05$, Fig. 5a). Compared to treatment with control or vehicle, treatment with SKLB325 significantly suppressed tumor growth, while no significant difference was observed in tumor weight between the 
A

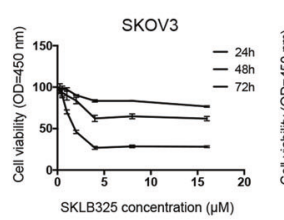

A2780s
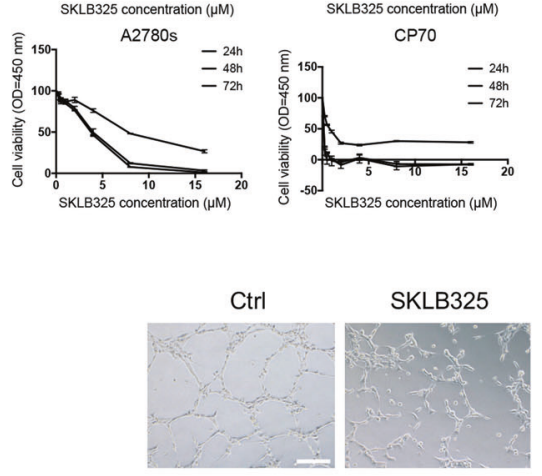

E
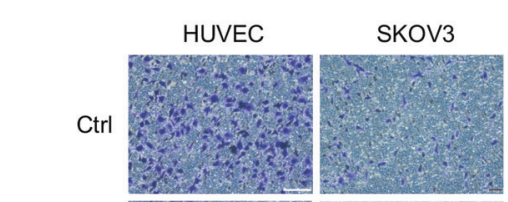

SKLB325
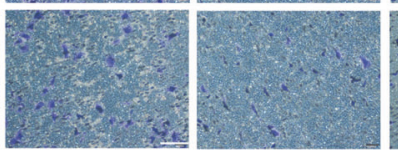

F

JMJD6
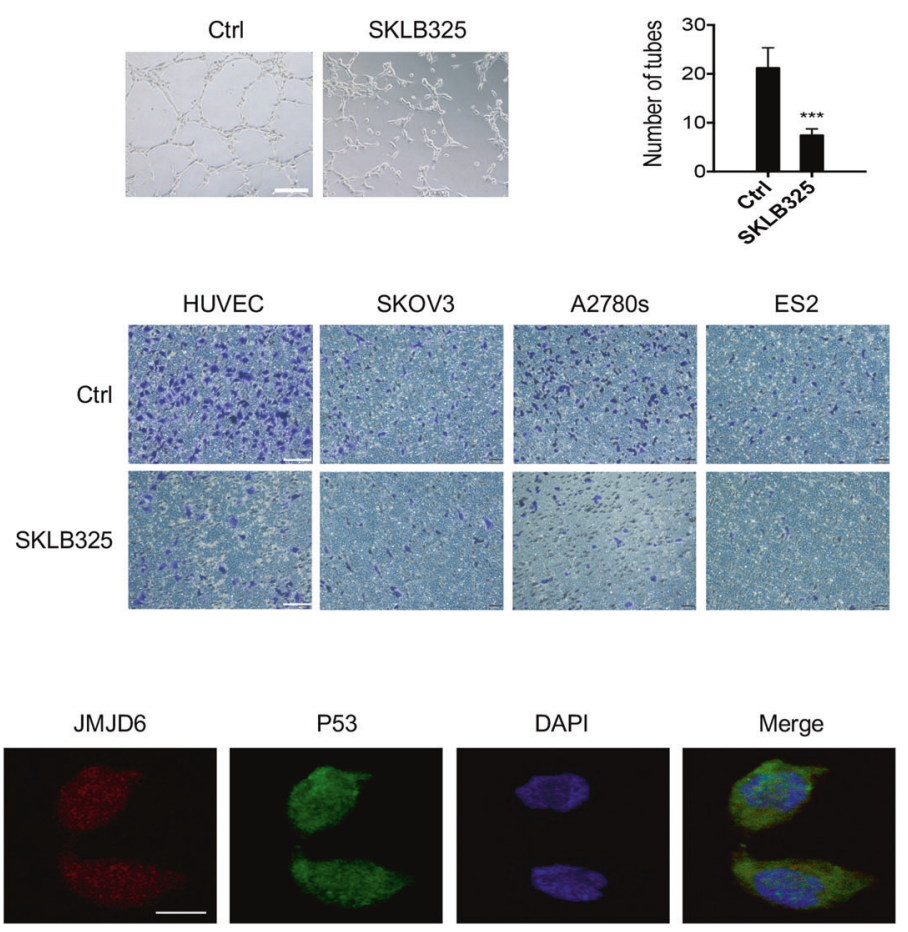
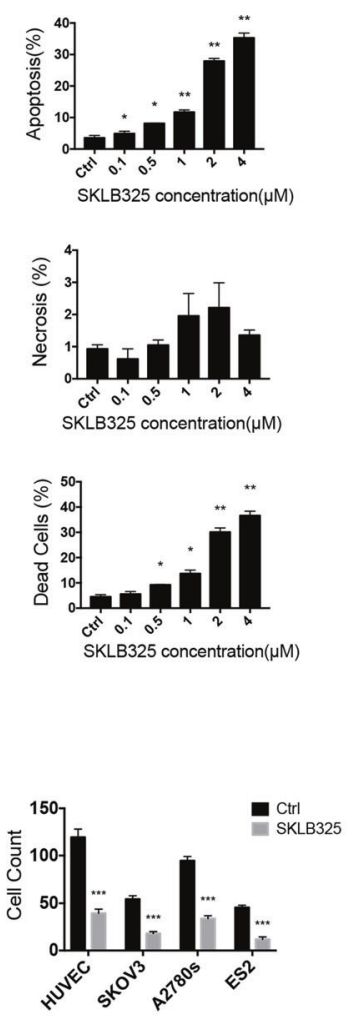

H

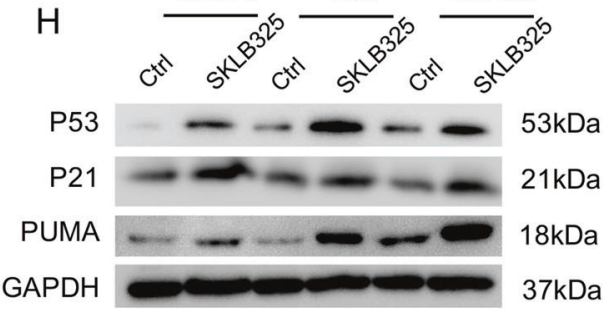

G

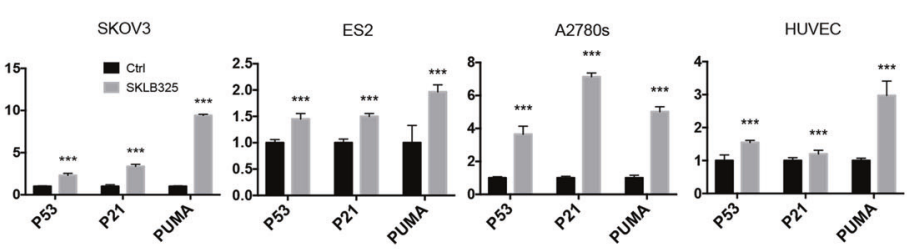

I

Ctrl SKLB325

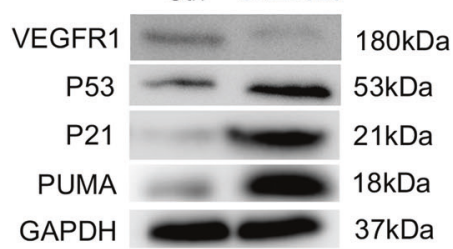

Fig. 4 Antitumor effects of SKLB325 in vitro. a The effect of SKLB325 on the proliferation of SKOV3, ES2, A2780s and CP70 cells was determined by CCK- 8 assays. Absorbances $(450 \mathrm{~nm})$ of cells cultured for $24 \mathrm{~h}, 48 \mathrm{~h}$, and $72 \mathrm{~h}$ in the presence of various concentrations of SKLB325 $(0,0.25,0.5,1,2,4,8$, and $16 \mu \mathrm{M})$ were determined. Each drug concentration was tested in quadruplicate. The cell viability data (mean \pm SD) are presented as percentages calculated by the following formula: Viability=1- (SKLB325-vehicle)/(control-blank). b, c Representative analyses of apoptotic, necrotic and dead cells induced by various concentrations $(0.1,0.5,1,2,4 \mu \mathrm{M})$ of SKLB325 in vitro were performed with flow cytometry with Annexin-V and PI staining. SKOV3 cells were treated with SKLB325 for $72 \mathrm{~h}$. Percentages of apoptotic, necrotic and dead cells are shown. d HUVECs were seeded in 24-well culture plates precoated with Matrigel. Magnification: $\times 100$, scale bar, $100 \mu \mathrm{m}$. e Migration of HUVECs, SKOV3, A2780s and ES2 cells was determined in chambers. Magnification: $\times 100$, scale bar, $100 \mu \mathrm{m}$. $\mathbf{f} \mathrm{JMJD} 6$ is physically associated with p53 in vitro. Immunofluorescence-stained endogenous JMJD6 (red) and p53 (green) were visualized by confocal microscopy. DAPI staining was included to visualize the cell nucleus (blue). Scale bar, $50 \mu \mathrm{m}$. g Measurement of mRNA levels of p53, p21 and PUMA by real-time RT-PCR. RT-PCR of CDNA obtained from SKOV3, ES2, A2780s, and HUVECs treated with or without SKLB325 for $72 \mathrm{~h}$. $\mathbf{h}$ Measurement of p53, p21 and PUMA protein levels by western blotting. Western blot of protein abstracts obtained from SKOV3, ES2 and A2780s cells treated with or without SKLB325 for $72 \mathrm{~h}$. i VEGFR1 protein levels were significantly downregulated after treatment with SKLB325 in HUVECs. p53, p21 and PUMA protein levels were significantly upregulated in HUVECs. The SKLB325 concentration was $4 \mu$ M. Data are presented as the mean values and standard deviations (SD). Each bar represents the mean \pm SD for quadruple or triplicate experiments. For statistical comparisons, each treated group was compared with the control groups using Student's $t$ test. ${ }^{*} p<0.05$, ${ }^{* *} p<0.01$ 
A

B
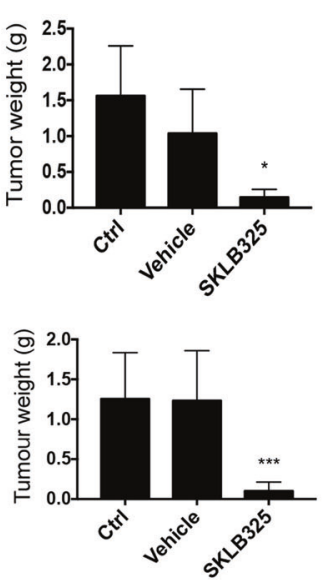

C

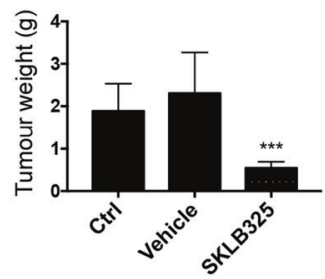

$\mathrm{D}$
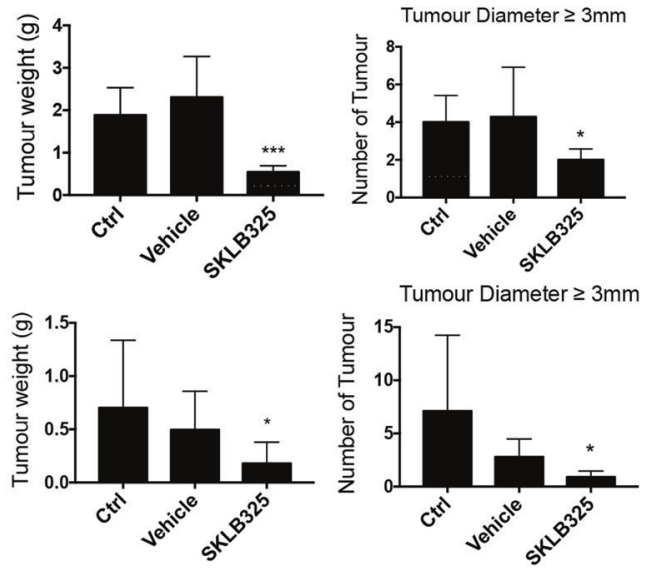

$$
\text { Tumour Diameter } \geq 3 \mathrm{~mm}
$$

$\mathrm{E}$

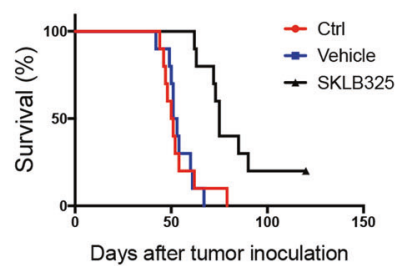

G

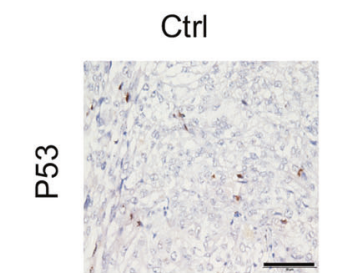

$\mathrm{F}$

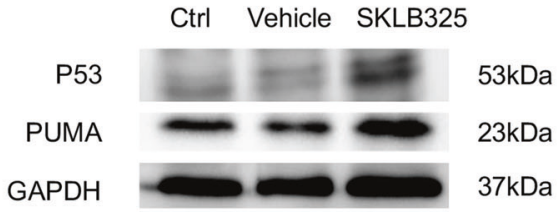

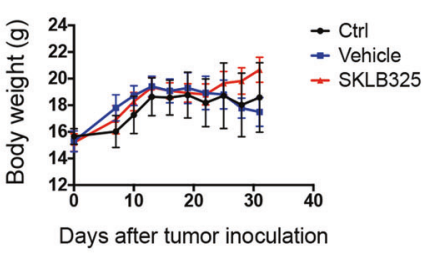
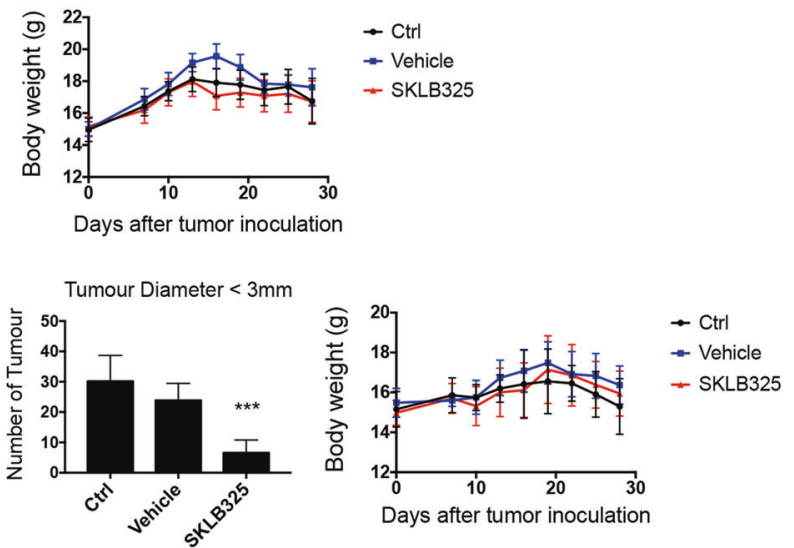

$$
\text { Tumour Diameter }<3 \mathrm{~mm}
$$
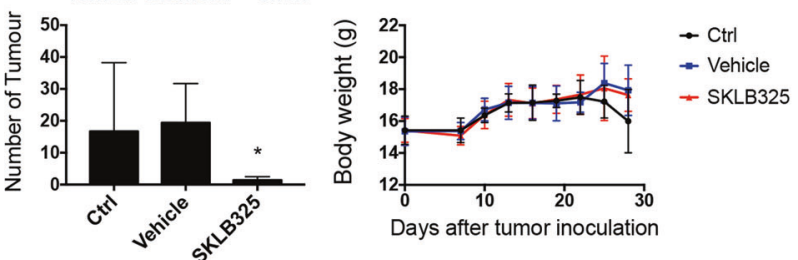

Vehicle
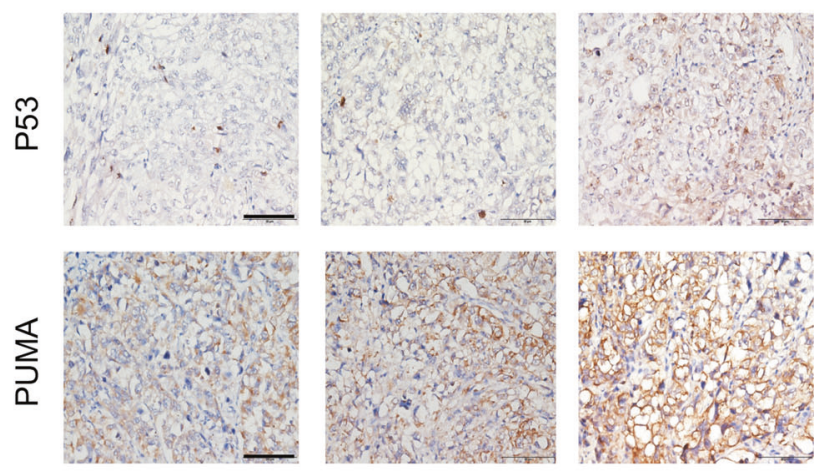

Fig. 5 SKLB325 has strong therapeutic effects on intraperitoneal dissemination xenograft mouse models. a SKLB325 has strong therapeutic effects on a SKOV3 intraperitoneal dissemination xenograft mouse model: Intraperitoneal tumor weight of each group; number of tumors $\geq 3 \mathrm{~mm}$ of each group; mice treated with SKLB325 showed no toxicity-dependent weight loss. b SKLB325 has strong therapeutic effects on an ES2 intraperitoneal dissemination xenograft mouse model: Ascites volume of each group; intraperitoneal tumor weight of each group; mice treated with SKLB325 showed no toxicity-dependent weight loss. c SKLB325 has strong therapeutic effects on a CP70 intraperitoneal dissemination xenograft mouse model: Number of tumours that were $\geq 3 \mathrm{~mm}$ in each group; Number of tumours that were $\leq 3 \mathrm{~mm}$ in each group; Intraperitoneal tumor weight of each group; Mice treated with SKLB325 showed no toxicity-dependent weight loss. d SKLB325 has strong therapeutic effects on an A2780s intraperitoneal dissemination xenograft mouse model: Number of tumors that were $\geq 3 \mathrm{~mm}$ in each group; Number of tumors that were $\leq 3 \mathrm{~mm}$ in each group; Intraperitoneal tumor weight of each group; Mice treated with SKLB325 showed no toxicity-dependent weight loss. Values are the mean \pm SD; $n=5-7$ mice/group. ${ }^{*} p<0.05,{ }^{* *} p<0.01,{ }^{* * *} p<0.001$, SKLB325 group versus control and vehicle groups, respectively. Control: without any treatment; Vehicle: the same dose of DMSO accompanied with NS. e Kaplan-Meier survival curve for tumor-bearing mice treated with control, vehicle, or SKLB325 using the SKOV3 intraperitoneal dissemination xenograft mouse model. Two mice in the SKLB325-treated group survived the entire 120 days after i.p. inoculation, when the planned experimental period ended. Survival was significantly longer in the mice treated with SKLB325 compared with the control and vehicle groups (log-rank test, $p=0.000)$, and there was no significant difference between the control and vehicle groups $(p>0.05)$. The median survival of the SKLB325 group was 75 days versus 51 days and 52 days in the control and vehicle groups, respectively; $n=10$ for each group. $\mathbf{f}$ Measurement of protein levels of P53 and PUMA by western blotting in vivo on the SKOV3 intraperitoneal dissemination xenograft mouse model. $\mathbf{g}$ Immunohistochemistry of p53 and PUMA in tumor tissue in the SKOV3 intraperitoneal dissemination xenograft mouse model. Magnification: $\times 200$; scale bar, $50 \mu \mathrm{m}$ 
control and vehicle groups $(p=0.108)$. The number of tumors whose diameter was $\geq 3 \mathrm{~mm}$ in the SKLB325 group was reduced compared to the two control groups (Fig. 5a). Similar results are shown in Fig. 5b-d. In the face of limitations by institutional ethics, the results of an animal study still indicated that the SKLB325 treatment protocols were effective in suppressing SKOV3, ES2, CP70, and A2780s tumor growth in nude mice.

There were no abnormalities visible to the naked eye observed in mice treated with SKLB325. No significant differences in body weight were found among the three groups (Fig. 5a-d). In addition to reduced cell proliferation and increased apoptosis, survival time also reflected the beneficial effects of SKLB325 on this human ovarian cancer model. As shown in the Kaplan-Meier survival curve in Fig. 5e, mice in the SKLB325 group lived longer than those in the two control groups, with a median survival of 75 days compared to 51 days and 52 days. No statistically significant difference in survival was observed between the control and vehicle groups $(p>0.05)$. At 120 days after i.p. inoculation, two mice in the SKLB325-treated group still survived when the experiment ended. These results demonstrated significant advantages in the SKLB325-treated group $(p<0.001)$.

SKLB325 upregulates the expression of p53 as well as its downstream effectors at the protein level in vivo

To detect the expression of p53 and its downstream effectors, western blotting was performed. As shown in Fig. $5 f$, the protein expression of p53 and PUMA was increased. Immunohistochemical staining in tumor tissues further demonstrated the promotion of p53 and PUMA in the inhibitor-treated tumours, as shown in Fig. 5g, Supplementary Figs. 4 and 5. Together, these results indicated that p53 and PUMA were induced after treatment with SKLB325 in vivo.

\section{SKLB325 inhibits cell proliferation and induces cell apoptosis in vivo}

To further confirm cell proliferation and apoptosis in vivo, we measured their effects by Ki-67 and TUNEL staining, as well as flow cytometry with Annexin-V and PI staining. The percentage of Ki67-positive cells was significantly reduced in the SKLB325 group compared to the control and vehicle groups ( $25.11 \pm 1.27$ versus $60.20 \pm 1.66$ and $64.19 \pm 1.09$, respectively, ${ }^{* *} p<0.001$, Fig. $\left.6 a, b\right)$. Many strongly positive nuclei were found in SKLB325-treated tumor tissues, as determined by immunofluorescence microscopy of TUNEL staining; however, such nuclei were rare in tumor tissues of the control groups $(28.23 \pm 5.07$ versus $8.90 \pm 2.19$ and $7.28 \pm$ 1.52 , respectively, ${ }^{* * *} p<0.001$, Fig. 6a, b). Furthermore, flow cytometry with Annexin-V and PI staining was used to investigate apoptosis of intraperitoneal cells in peritoneal lavage fluid. The results showed that intraperitoneal apoptotic cells $(27.62 \pm 1.90 \%$ versus $14.45 \pm 2.21 \%$ and $9.59 \pm 2.12 \%$, respectively, ${ }^{* *} p<0.01$, Fig. $6 c)$ and dead cells $(42.08 \pm 2.82 \%$ versus $24.00 \pm 2.15 \%$ and $22.97 \pm 4.60 \%$, respectively, ${ }^{* *} p<0.01$, Fig. $6 \mathrm{~d}$ ) were remarkably increased in mice treated with SKLB325 compared to the control and vehicle groups. As shown in Fig. 6e, in histopathological examination of tumor tissues stained with $\mathrm{H} \& \mathrm{E}$, we detected no obvious necrotic or apoptotic cells in the tumours isolated from mice with control or vehicle treatment, but tumours isolated from mice in the SKLB325 group exhibited large areas of necrotic or apoptotic cells.

Inhibition of angiogenesis by SKLB325 in vivo

As the antiangiogenic effect of SKLB325 was found in vitro, we used immunohistochemical staining of VEGF and CD31, which had highly specific affinity for vascular endothelial cells, for further study. We measured microvessel numbers in endothelial cells that were stained by VEGF and CD31 and evaluated tumor sections for VEGF and CD31 staining. The number of cells stained by VEGF was significantly reduced in tumors treated with SKLB325 (17.10
2.21) compared with the control and vehicle groups ( $40.97 \pm 1.13$ and $49.49 \pm 1.56$, respectively, ${ }^{* * *} p<0.001$, Fig. $6 f$, g). Similarly, the number of microvessels marked by CD31 was also significantly reduced in tumours treated with SKLB325 (17.00 \pm 1.41$)$ compared with the two control groups $\left(68.40 \pm 2.14\right.$ and $68.60 \pm 1.97,{ }^{* * *} p<$ 0.001 , Fig. $6 f, g)$. These results demonstrated that angiogenesis within this intraperitoneal carcinomatosis model was inhibited in these mice of the SKLB325 group, suggesting that the antitumor activity of the inhibitor was due, at least in part, to inhibition of angiogenesis in our tumor model.

No noticeable adverse effects were found in the intraperitoneal dissemination xenograft mouse mode

Histological examination of the heart, spleen, kidney, lung, and liver revealed no significant differences among all groups (Fig. 7a). These results further indicated that SKLB325 treatment did not cause obvious adverse effects at the indicated doses and times in vivo, and the mice showed good tolerance of the current treatment procedures. Routine analysis and biochemical analysis of blood from all experimental groups and a normal group with no treatment were collected. White blood cells (WBC), red blood cells (RBC), hemoglobin (HGB), and platelets (PLT) exhibited no significant differences among these four groups (Fig. 7b). Furthermore, there were no significant differences in alanine transaminase (ALT), aspartate transaminase (AST), albumin (ALB), alkaline phosphatase (ALP), total protein (TP), cholesterol (CHOL), urea, uric acid (UA), blood urea nitrogen (BUN), or glucose (GLU) among the normal, control, vehicle, and SKLB325 groups (Fig. 7c).

\section{DISCUSSION}

Mortality of ovarian cancer remains extraordinarily high, despite many improvements in treatments for patients with ovarian cancer, including surgery, radiotherapy, chemotherapy, and new biological therapies. ${ }^{5-7}$ Furthermore, $60-70 \%$ of patients with ovarian cancer who respond to standard primary treatment relapse or die within 5 years after initial diagnosis. ${ }^{8,47}$ This terrible situation has stimulated the development of new strategies to improve therapeutic efficacy.

Recent studies indicate that ablation of the JMJD6 gene in $C$. elegans delays the engulfment of apoptotic cells, 15,37 and depletion of JMJD6 promoted apoptosis, induced cell death by sensitizing cells to DNA damaging reagent, and repressed cell tumorigenesis and proliferation, which is mediated by $\mathrm{p} 53 .{ }^{9}$ The expression of JMJD6 in some human cancers is evidently upregulated, such as lung adenocarcinoma, ${ }^{39}$ breast ductal carcinomas, ${ }^{40}$ and colon adenocarcinomas. ${ }^{9}$ Furthermore, high expression of JMJD6 is related to tumor growth, tumor metastasis, and high tumor pathological classification, ${ }^{9,38-41}$ indicating that JMJD6 may act as a novel therapeutic target for cancer intervention. However, there is still limited information on the expression of JMJD6 in ovarian cancer. Furthermore, no JMJD6 inhibitors have been developed and applied to the research of ovarian cancer.

In our research, JMJD6 protein expression was detected by immunohistochemical staining in a cohort of 146 ovarian cancer specimens, and we analyzed the relationship between JMJD6 protein expression and clinicopathological variables. The results indicated that high expression of JMJD6 in ovarian cancer was significantly associated with age, clinical stage, pT status, pN status, and relapse. Patients with high JMJD6 expression had significantly worse OS and DFS. These results clearly demonstrated that high expression of JMJD6 was associated with adverse clinical outcomes of ovarian cancer. In view of the above results, we demonstrated that JMJD6 is important in ovarian cancer and may provide a novel therapeutic target.

Our study investigated whether SKLB325 inhibition could be a novel therapeutic approach to combat ovarian cancer by 
A
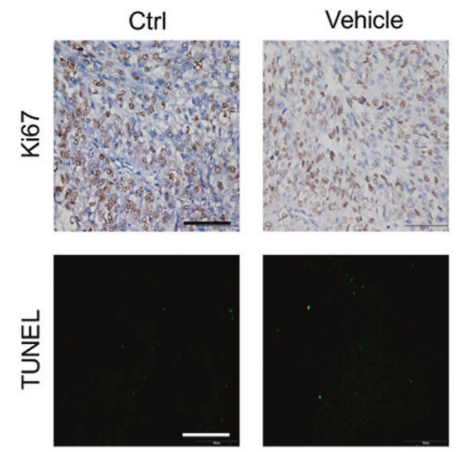

C

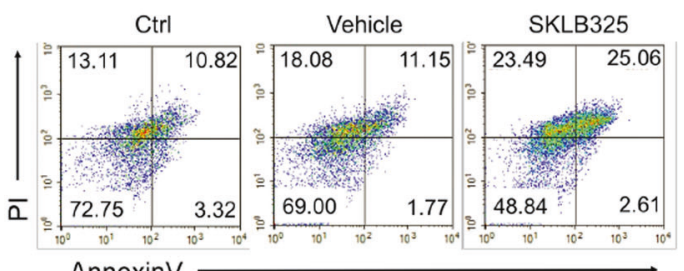

E
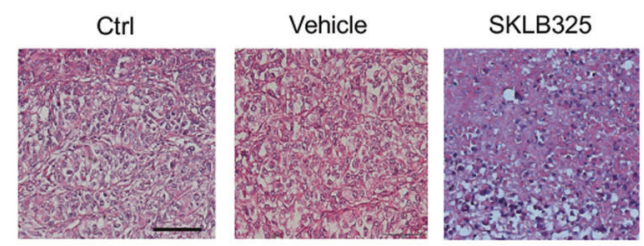

G
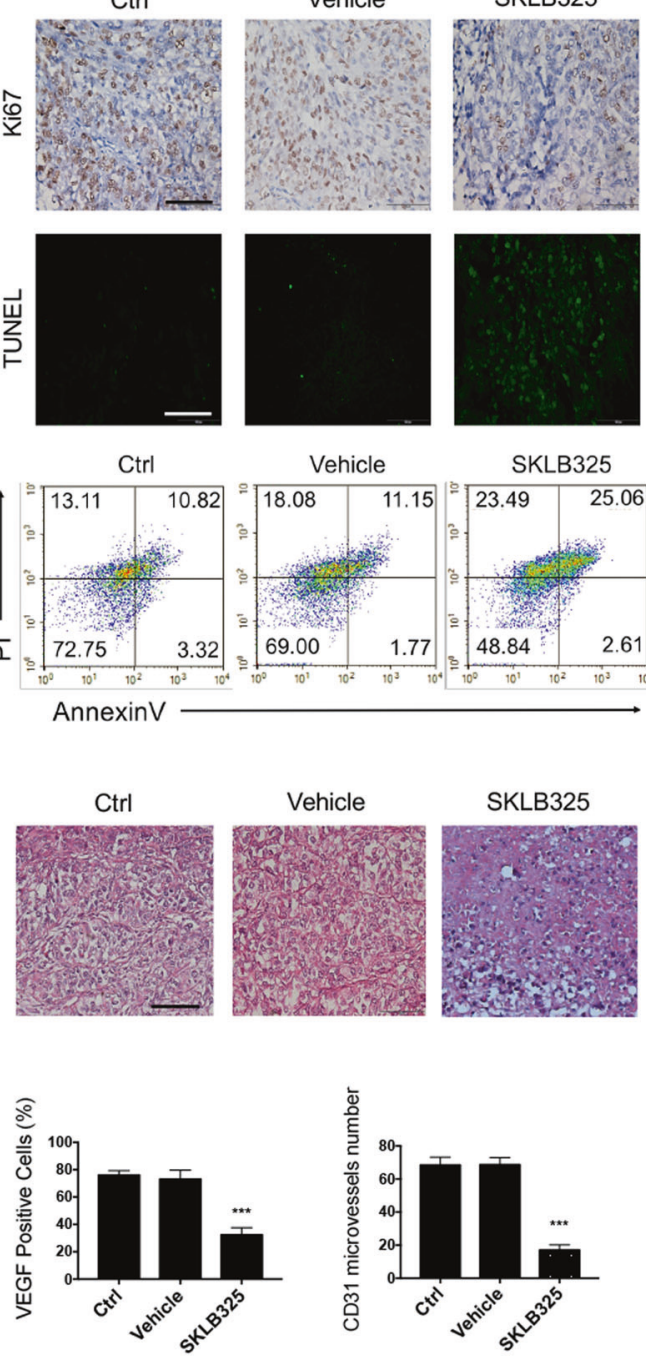

B

D
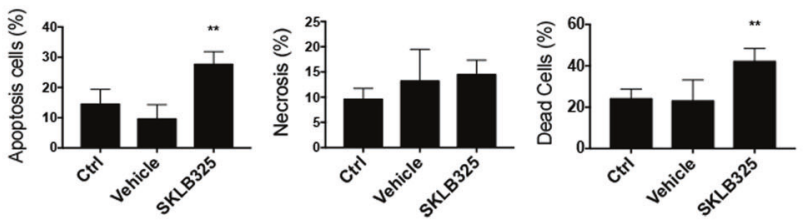

$\mathrm{F}$
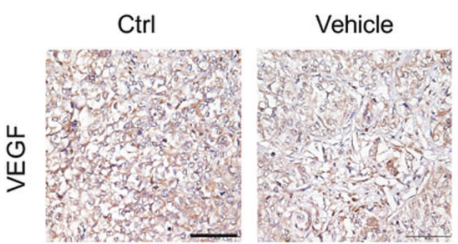

SKLB325
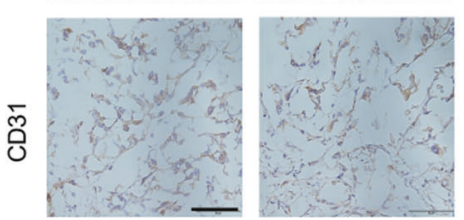

Fig. 6 SKLB325 induces apoptosis and suppresses angiogenesis in vivo. a Representative images for immunohistochemical staining of Ki-67 and TUNEL on SKOV3 tumors isolated from mice treated with control, vehicle and SKLB325. The data showed that SKLB325 can lead to suppression of tumor cell proliferative activity (scale bar, $50 \mu \mathrm{m}$ ) and promotion of tumor apoptotic activity (scale bar, $100 \mu \mathrm{m})$. b Percentages of Ki-67-positive nuclei apparently decreased, and percentages of TUNEL-positive nuclei apparently increased. The number of Ki-67-positive or TUNEL-positive cells was counted in five different fields under a light microscope at $\times 200$ magnification, ${ }^{* * *} p<0.001$. The results are presented as the mean \pm SD $(n=5)$ versus control and vehicle groups. c Representative images for flow cytometry with Annexin-V and PI staining of peritoneal lavage fluid showed intraperitoneal apoptotic cells increased in the mice that were treated with SKLB325. $\mathbf{d}$ Percentages of total apoptotic cells, necrotic and dead cells are shown. Each bar represents the mean \pm SD $(n=5) ;{ }^{* *} p<0.01$. e Representative images of hematoxylin and eosin (H \& E) staining of intraperitoneal tumours isolated from mice treated with control, vehicle or SKLB325. No obvious necrotic cells were found in the tumors isolated from mice with control or vehicle treatment, but tumours isolated from mice with SKLB325 treatment exhibited large areas of necrotic or apoptotic cells. Magnification: $\times 100$; scale bar, $100 \mu \mathrm{m}$. f Representative images of immunohistochemical VEGF and CD31 staining. Magnification: $\times 100$; scale bar, $100 \mu \mathrm{m}$. The quantification of MVD was estimated by counting the number of microvessels in five random fields at $\times 200$ magnification. $\mathbf{g}$ Immunostaining of VEGF on tumor tissue. A single positively stained cell or single microvessel was defined as a discrete cluster for CD31. The SKLB325-treated group showed reduced stained cells and microvessel density. Data are presented as the mean \pm SD $(n=5)$. ${ }^{* *} p<0.001$ versus the other two groups; control group: without any treatment; vehicle group: the same dose of DMSO accompanied with NS

repressing cell proliferation and tumorigenesis. Several observations have been made in this study concerning the antitumor effects of SKLB325 on ovarian cancer. The inhibitor SKLB325 exerted a cytostatic effect, the results of the CCK- 8 cell proliferation assay indicated that SKLB325 could suppress cell proliferation in vitro, and this treatment led to inhibition of cell proliferation in a significant dose-dependent manner. Furthermore, establishment of a peritoneal carcinomatosis model in $\mathrm{BALB} / \mathrm{C}$ nude mice provided evidence that JMJD6 inhibition had strong therapeutic effects on an intraperitoneal dissemination xenograft mouse model. Tumor volumes in the SKLB325 group were notably reduced compared with the two control groups, which was in agreement with extensive reports that have supported the role of JMJD6 in carcinogenesis., ${ }^{9,39}$ SKLB325 prolonged the survival of tumor-bearing mice. The results showed no noticeable adverse effects in the intraperitoneal dissemination xenograft mouse mode and provided evidence for the safety of the inhibitor SKLB325.

Many studies have shown that inducing apoptosis and inhibiting angiogenesis are possible mechanisms of the inhibitor SKLB325. 


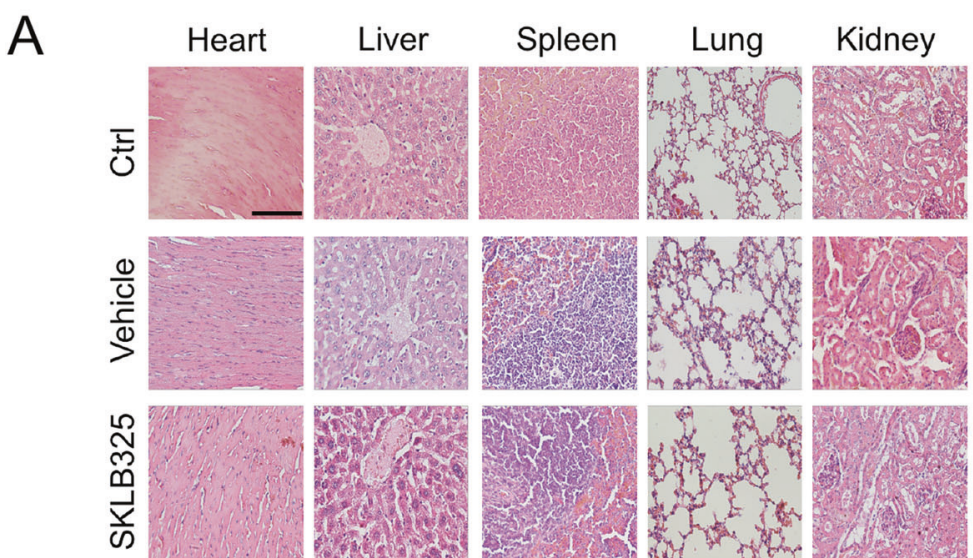

C
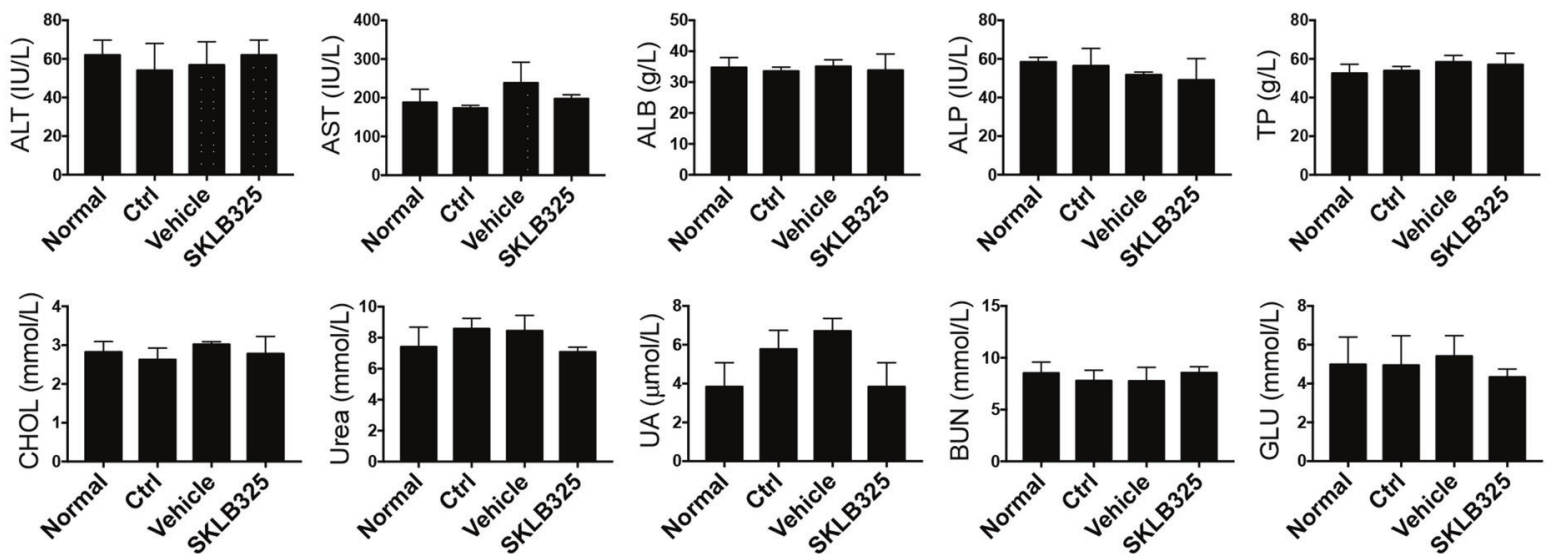

Fig. 7 No noticeable adverse effects were found in the intraperitoneal dissemination xenograft mouse model. a Histological staining of heart, liver, spleen, lung, and kidney from mice treated with SKLB325 revealed no significant pathologic differences $(n=5)$. Magnification: $\times 200$; scale bar, $50 \mu \mathrm{m}$. b The results of WBC, RBC, HGB and PLT in the normal group, control group, vehicle group and SKLB325 group exhibited no significant differences among these four groups. c Results of ALT, AST, ALB, ALP, TP, CHOL, urea, UA, BUN, and GLU among the normal, control, vehicle and SKLB325 groups exhibited no significant differences in these four groups

Our flow cytometry and $\mathrm{Pl} /$ Annexin $\mathrm{V}$ results indicated that the inhibitor SKLB325 induced apoptosis and cell death. Recent studies showing that JMJD6 could increase proliferation in MCF-7 cells (breast adenocarcinoma cells) also supported our results. ${ }^{40}$ Angiogenesis is a key factor in the development of metastatic carcinoma, $^{48}$ and many previous studies have proven that angiogenesis is necessary for tumorigenesis and progression, ${ }^{49-51}$ which is required for tumor formation, as well as for delivering oxygen and nutrients. ${ }^{52}$ CD31 and VEGF, markers of angiogenesis, have been identified as prognostic indicators in ovarian cancer. ${ }^{53-57}$ The Matrigel endothelial tube formation assay suggested that SKLB325 could contribute to inhibiting angiogenesis in HUVECs in vitro, which was consistent with a previous study showing that JMJD6 silencing could affect the splicing of angiogenesis-related genes (VEGF receptor 1) in ECs and that it was necessary for angiogenic sprouting. ${ }^{58}$ Furthermore, we found that $\mathrm{p} 53$ protein colocalized with JMJD6 in the nucleus and that SKLB325 could upregulate $\mathrm{p} 53$ and the expression of downstream effectors at both the mRNA and protein levels in vitro. Similar to previous research, JMJD6 had a negative effect on the p53 pathway, and depletion of JMJD6 promoted apoptosis, induced cell death, and repressed cell tumorigenesis and proliferation, which is mediated by $\mathrm{p} 53 .{ }^{9}$ Consistent with the results in vitro, staining of Ki-67, TUNEL analysis and flow cytometry with Annexin-V and PI indicated that SKLB325 could inhibit cell proliferation and induce cell apoptosis in vivo. Immunohistochemical staining also suggested that SKLB325 could upregulate $\mathrm{p} 53$ and its downstream effectors. We found inhibition of angiogenesis by SKLB325 in vivo as detected by immunohistochemical staining of VEGF and CD31, which coincided with previous studies. ${ }^{58}$ Although no studies on the antitumor effects of JMJD6 inhibitors have been published, previous studies have reported that tumor growth was obviously impaired in BALB/C nude mice that received JMJD6-depleted HCT116 p53+/+tumor transplants, and the literature supports the probability that inhibition of JMJD6 may have antitumor effects on human cancers. ${ }^{9}$ However, there are still some shortcomings in our experiment; for example, JMJD6 depletion or p53 depletion experiments may confirm that SKLB325 suppresses cancer progression through inhibition of JMJD6 and upregulation of $\mathrm{p} 53$ expression. In addition, the inhibition of the enzymatic activity of JMJD6 and other family members of JmjC domain-containing proteins by the inhibitor SKLB325 should be performed in future studies.

\section{CONCLUSIONS}

In the present study, we demonstrated that JMJD6 may be a marker of poor prognosis in ovarian cancer. Furthermore, we found that the inhibitor SKLB325 suppresses ovarian cancer growth through inhibition of proliferation and induction of apoptosis and cell death, and inhibiting angiogenesis may play a significant role in inhibiting tumor growth. SKLB325 significantly prolongs the survival of tumor-bearing mice without obvious side effects. These results suggest that SKLB325 may be a potential candidate drug for treatment of ovarian cancer. 


\section{DATA AVAILABILITY}

All data generated or analyzed during this study are included in this published article.

\section{ACKNOWLEDGEMENTS}

This work is supported by the National Key Research and Development Program of China (No. 2016YFA0201402), the National Major Scientific and Technological Special Project for "Significant New Drugs Development" (No. 2018ZX09733001) and the Natural Science Foundation of China (No. 81602492)

\section{AUTHOR CONTRIBUTIONS}

X.W., H.Z. and Y.T. designed and performed the experiments and wrote the paper. S. H., Y.Q., X.W., R.P. and S.C. performed the experiments. X.L. wrote the paper. T.Y. provided cells and performed experiments. Y.P., S.Y. and X.Z. designed experiments and supervised the project. Z.F. and X.W. performed experiments and wrote the paper. All authors discussed the results and contributed to the paper.

\section{ADDITIONAL INFORMATION}

The online version of this article (https://doi.org/10.1038/s41392-019-0055-8) contains supplementary material, which is available to authorized users

Competing interests: The authors declare no competing interests.

Ethical approval: All animal studies were approved by the Institutional Animal Care and Use Committee of Sichuan University in accordance with all regulations and guidelines of China. HUVEC isolation was approved by the institutional review board of the Medical Faculty at the West China Second Hospital, Sichuan University.Consent for publicationWe all consent to publication.

\section{REFERENCES}

1. Siegel, R. L., Miller, K. D. \& Jemal, A. Cancer statistics, 2016. CA Cancer J. Clin. 66, 7-30 (2016).

2. Prat, J. FIGO's staging classification for cancer of the ovary, fallopian tube, and peritoneum: abridged republication. J. Gynecol. Oncol. 26, 87-89 (2015).

3. Jayson, G. C., Kohn, E. C., Kitchener, H. C. \& Ledermann, J. A. Ovarian cancer. Lancet (London, England) 384, 1376-1388 (2014).

4. Lin, X. et al. RNA-binding protein LIN28B inhibits apoptosis through regulation of the AKT2/FOXO3A/BIM axis in ovarian cancer cells. Signal Trans. Targeted Ther. $\mathbf{3}$ 23 (2018).

5. Liu, J. \& Matulonis, U. A. New strategies in ovarian cancer: translating the molecular complexity of ovarian cancer into treatment advances. Clin. Cancer Res.: Off. J. Am. Assoc. Cancer Res. 20, 5150-5156 (2014).

6. Robella, M. et al. Treatment of peritoneal carcinomatosis from ovarian cancer by surgical cytoreduction and hyperthermic intraperitoneal chemotherapy (HIPEC). Minerva Chir. 69, 27-35 (2014).

7. Zsiros, E., Tanyi, J., Balint, K. \& Kandalaft, L. E. Immunotherapy for ovarian cancer: recent advances and perspectives. Curr. Opin. Oncol. 26, 492-500 (2014).

8. Cardenas, C., Alvero, A. B., Yun, B. S. \& Mor, G. Redefining the origin and evolution of ovarian cancer: a hormonal connection. Endocr. Relat. Cancer 23, R411-R422 (2016).

9. Wang, F. et al. JMJD6 promotes colon carcinogenesis through negative regulation of p53 by hydroxylation. PLoS Biol. 12, e1001819 (2014).

10. Unoki, M. et al. Lysyl 5-hydroxylation, a novel histone modification, by Jumonji domain containing 6 (JMJD6). J. Biol. Chem. 288, 6053-6062 (2013).

11. Guo, G. \& Cui, Y. New perspective on targeting the tumor suppressor p53 pathway in the tumor microenvironment to enhance the efficacy of immunotherapy. J. Immunother. Cancer 3, 9 (2015).

12. Kim, J. H. et al. p53 acetylation enhances Taxol-induced apoptosis in human cancer cells. Apoptosis 18, 110-120 (2013).

13. Parrales, A. \& Iwakuma, T. Targeting oncogenic mutant p53 for cancer therapy. Front. Oncol. 5, 288 (2015).

14. Hong, B., van den Heuvel, A. P., Prabhu, V. V., Zhang, S. \& El-Deiry, W. S. Targeting tumor suppressor p53 for cancer therapy: strategies, challenges and opportunities. Curr. Drug Targets 15, 80-89 (2014).

15. Wang, $X$. et al. Cell corpse engulfment mediated by $C$. elegans phosphatidylserine receptor through CED-5 and CED-12. Science 302, 1563-1566 (2003).

16. Bose, J. et al. The phosphatidylserine receptor has essential functions during embryogenesis but not in apoptotic cell removal. J. Biol. 3, 15 (2004).

17. Fadok, V. A. et al. A receptor for phosphatidylserine-specific clearance of apoptotic cells. Nature 405, 85-90 (2000).
18. Hahn, P. et al. Analysis of Jmjd6 cellular localization and testing for its involvement in histone demethylation. PloS ONE 5, e13769 (2010).

19. Tibrewal, N., Liu, T., Li, H. \& Birge, R. B. Characterization of the biochemical and biophysical properties of the phosphatidylserine receptor (PS-R) gene product Mol. Cell. Biochem. 304, 119-125 (2007).

20. Hahn, P., Bose, J., Edler, S. \& Lengeling, A. Genomic structure and expression of Jmjd6 and evolutionary analysis in the context of related JmjC domain containing proteins. BMC Genom. 9, 293 (2008).

21. Cui, P., Qin, B., Liu, N., Pan, G. \& Pei, D. Nuclear localization of the phosphatidylserine receptor protein via multiple nuclear localization signals. Exp. Cell Res. 293, 154-163 (2004).

22. Cikala, M. et al. The phosphatidylserine receptor from Hydra is a nuclear protein with potential Fe(II) dependent oxygenase activity. BMC Cell Biol. 5, 26 (2004).

23. Wolf, A., Schmitz, C. \& Bottger, A. Changing story of the receptor for phosphatidylserine-dependent clearance of apoptotic cells. EMBO Rep. 8 465-469 (2007).

24. Mitchell, J. E. et al. The presumptive phosphatidylserine receptor is dispensable for innate anti-inflammatory recognition and clearance of apoptotic cells. J. Biol. Chem. 281, 5718-5725 (2006).

25. Zakharova, L., Dadsetan, S. \& Fomina, A. F. Endogenous Jmjd6 gene product is expressed at the cell surface and regulates phagocytosis in immature monocytelike activated THP-1 cells. J. Cell. Physiol. 221, 84-91 (2009).

26. Webby, C. J. et al. Jmjd6 catalyses lysyl-hydroxylation of U2AF65, a protein associated with RNA splicing. Science 325, 90-93 (2009).

27. Chang, B., Chen, Y., Zhao, Y. \& Bruick, R. K. JMJD6 is a histone arginine demethylase. Science 318, 444-447 (2007).

28. Loenarz, C. \& Schofield, C. J. Expanding chemical biology of 2-oxoglutarate oxygenases. Nat. Chem. Biol. 4, 152-156 (2008).

29. Hong, X. et al. Interaction of JMJD6 with single-stranded RNA. Proc. Natl Acad. Sci. USA 107, 14568-14572 (2010).

30. Bottger, A., Islam, M. S., Chowdhury, R., Schofield, C. J. \& Wolf, A. The oxygenase Jmjd6-a case study in conflicting assignments. Biochem. J. 468, 191-202 (2015).

31. McNeill, L. A. et al. The use of dioxygen by HIF prolyl hydroxylase (PHD1) Bioorganic Med. Chem. Lett. 12, 1547-1550 (2002).

32. Rose, N. R., McDonough, M. A., King, O. N., Kawamura, A. \& Schofield, C. J. Inhibition of 2-oxoglutarate dependent oxygenases. Chem. Soc. Rev. 40, 4364-4397 (2011).

33. Chowdhury, R. et al. The oncometabolite 2-hydroxyglutarate inhibits histone lysine demethylases. EMBO Rep.12, 463-469 (2011).

34. Liu, W. et al. Brd4 and JMJD6-associated anti-pause enhancers in regulation of transcriptional pause release. Cell 155, 1581-1595 (2013).

35. Heim, A. et al. Jumonji domain containing protein 6 (Jmjd6) modulates splicing and specifically interacts with arginine-serine-rich (RS) domains of SR- and SR-like proteins. Nucleic Acids Res. 42, 7833-7850 (2014)

36. Mantri M., et al. Crystal structure of the 2-oxoglutarate- and Fe(II)-dependent lysyl hydroxylase JMJD6. J. Mol. Biol., https://doi.org/10.1016/j.jmb.2010.05.054 (2010).

37. Neumann, B. et al. EFF-1-mediated regenerative axonal fusion requires components of the apoptotic pathway. Nature 517, 219-222 (2015).

38. Aprelikova, $\mathrm{O}$. et al. The epigenetic modifier JMJD6 is amplified in mammary tumors and cooperates with c-Myc to enhance cellular transformation, tumor progression, and metastasis. Clin. Epigenet. 8, 38 (2016).

39. Zhang, J., Ni, S. S., Zhao, W. L., Dong, X. C. \& Wang, J. L. High expression of JMJD6 predicts unfavorable survival in lung adenocarcinoma. Tumour Biol. 34 2397-2401 (2013).

40. Lee, Y. F. et al. JMJD6 is a driver of cellular proliferation and motility and a marker of poor prognosis in breast cancer. Breast Cancer Res. 14, R85 (2012).

41. Poulard, C. et al. Role of JMJD6 in breast tumourigenesis. PloS ONE 10, e0126181 (2015).

42. Chen, C. F. et al. Regulation of T cell proliferation by JMJD6 and PDGF-BB during chronic hepatitis B infection. Sci. Rep. 4, 6359 (2014).

43. Gyuris, J., Golemis, E., Chertkov, H. \& Brent, R. Cdi1, a human G1 and S phase protein phosphatase that associates with Cdk2. Cell 75, 791-803 (1993).

44. Lawrence, P. \& Rieder, E. Identification of RNA helicase $A$ as a new host factor in the replication cycle of foot-and-mouth disease virus. J. Virol. 83, 11356-11366 (2009).

45. Lawrence, P., Conderino, J. S. \& Rieder, E. Redistribution of demethylated RNA helicase A during foot-and-mouth disease virus infection: role of Jumonji Cdomain containing protein 6 in RHA demethylation. Virology 452-453, 1-11 (2014).

46. Fang, Z. et al. Discovery of pyrazolo[1,5-a]pyrimidine-3-carbonitrile derivatives as a new class of histone lysine demethylase 4D (KDM4D) inhibitors. Bioorganic Med. Chem. Lett. 27, 3201-3204 (2017).

47. Siegel, R., Ma, J., Zou, Z. \& Jemal, A. Cancer statistics, 2014. CA Cancer J. Clin. 64, 9-29 (2014). 
14

48. Carmeliet, P. Angiogenesis in life, disease and medicine. Nature 438, 932-936 (2005).

49. Mittal, K., Ebos, J. \& Rini, B. Angiogenesis and the tumor microenvironment: vascular endothelial growth factor and beyond. Semin. Oncol. 41, 235-251 (2014).

50. Kubota, Y. Tumor angiogenesis and anti-angiogenic therapy. Keio J. Med. 61, 47-56 (2012).

51. Nussenbaum, F. \& Herman, I. M. Tumor angiogenesis: insights and innovations. J. Oncol. 2010, 132641 (2010).

52. Teodoro, J. G., Evans, S. K. \& Green, M. R. Inhibition of tumor angiogenesisbyp53: a new role for the guardian of the genome. J. Mol. Med. (Berlin, Germany) 85, 1175-1186 (2007)

53. Ebos, J. M. et al. Accelerated metastasis after short-term treatment with a potent inhibitor of tumor angiogenesis. Cancer Cell 15, 232-239 (2009).

54. Folkman, J. Angiogenesis: an organizing principle for drug discovery? Nat. Rev. Drug Disc. 6, 273-286 (2007).

55. Secord, A. A. et al. Co-expression of angiogenic markers and associations with prognosis in advanced epithelial ovarian cancer: a Gynecologic Oncology Group study. Gynecol. Oncol. 106, 221-232 (2007).

56. Rubatt, J. M. et al. Independent prognostic relevance of microvessel density in advanced epithelial ovarian cancer and associations between CD31, CD105, p53 status, and angiogenic marker expression: A Gynecologic Oncology Group study. Gynecol. Oncol. 112, 469-474 (2009).
57. Goodheart, M. J. et al. The relationship of molecular markers of p53 function and angiogenesis to prognosis of stage I epithelial ovarian cancer. Clin. Cancer Res. 11, 3733-3742 (2005)

58. Boeckel, J. N. et al. Jumonji domain-containing protein 6 (Jmjd6) is required for angiogenic sprouting and regulates splicing of VEGF-receptor 1. Proc. Natl Acad. Sci. USA 108, 3276-3281 (2011).

(i) Open Access This article is licensed under a Creative Commons Attribution 4.0 International License, which permits use, sharing, adaptation, distribution and reproduction in any medium or format, as long as you give appropriate credit to the original author(s) and the source, provide a link to the Creative Commons license, and indicate if changes were made. The images or other third party material in this article are included in the article's Creative Commons license, unless indicated otherwise in a credit line to the material. If material is not included in the article's Creative Commons license and your intended use is not permitted by statutory regulation or exceeds the permitted use, you will need to obtain permission directly from the copyright holder. To view a copy of this license, visit http://creativecommons. org/licenses/by/4.0/.

(c) The Author(s) 2019 